\title{
Loss of CaMKI Function Disrupts Salt Aversive Learning in C. elegans
}

\author{
Jana P. Lim, ${ }^{1,2,3 *}$ Holger Fehlauer, ${ }^{3 *}$ (1)Alakananda Das, ${ }^{3}$ @ Gabriella Saro, ${ }^{5}$ @Dominique A. Glauser, ${ }^{5}$ Anne Brunet, ${ }^{1,2,4}$ \\ and ${ }^{\circ}$ Miriam B. Goodman ${ }^{1,3}$ \\ ${ }^{1}$ Neurosciences Graduate Program, ${ }^{2}$ Department of Genetics, ${ }^{3}$ Department of Molecular and Cellular Physiology, ${ }^{4}$ Glenn Center for the Biology of Aging at \\ Stanford University, Stanford, California 94305, and ${ }^{5}$ Department of Biology, University of Fribourg, 1700 Fribourg, Switzerland
}

The ability to adapt behavior to environmental fluctuations is critical for survival of organisms ranging from invertebrates to mammals. Caenorhabditis elegans can learn to avoid sodium chloride when it is paired with starvation. This behavior may help animals avoid areas without food. Although some genes have been implicated in this salt-aversive learning behavior, critical genetic components, and the neural circuit in which they act, remain elusive. Here, we show that the sole worm ortholog of mammalian CaMKI/IV, CMK-1, is essential for salt-aversive learning behavior in C. elegans hermaphrodites. We find that CMK-1 acts in the primary salt-sensing ASE neurons to regulate this behavior. By characterizing the intracellular calcium dynamics in ASE neurons using microfluidics, we find that loss of $c m k-1$ has subtle effects on sensory-evoked calcium responses in ASE axons and their modulation by salt conditioning. Our study implicates the expression of the conserved CaMKI/CMK-1 in chemosensory neurons as a regulator of behavioral plasticity to environmental salt in C. elegans.

Key words: calcium imaging; CaM kinase; chemotaxis; learning; salt-seeking

\section{Significance Statement}

Like other animals, the nematode Caenorhabditis elegans depends on salt for survival and navigates toward high concentrations of this essential mineral. In addition to its role as an essential nutrient, salt also causes osmotic stress at high concentrations. A growing body of evidence indicates that $C$. elegans balances the requirement for salt with the danger it presents through a process called salt-aversive learning. We show that this behavior depends on expression of a calcium/calmodulin-dependent kinase, CMK-1, in the ASE salt-sensing neurons. Our study identifies CMK-1 and salt-sensitive chemosensory neurons as key factors in this form of behavioral plasticity.

\section{Introduction}

Behavioral plasticity allows animals to survive a constantly changing environment. This trait enables organisms to seek environments

Received June 9, 2017; revised April 16, 2018; accepted May 25, 2018.

Author contributions: M.B.G. edited the paper. J.P.L., H.F., A.B., and M.B.G. designed research; J.P.L., H.F., A.D., and D.A.G. performed research; G.S. and D.A.G. contributed unpublished reagents/analytic tools; J.P.L., H.F., A.D., D.A.G., and M.B.G. analyzed data; J.P.L., H.F., A.D., D.A.G., A.B., and M.B.G. wrote the paper.

This work was supported by a National Science Foundation Graduate Research Fellowship (J.P.L.), NIH Grant NS047715 (M.B.G.), NIH Grant AG031198 (A.B.), and Swiss National Science Foundation Grants BSSGI0_155764 and PP00P3_150681 (D.A.G.). We thank C. Murphy (Princeton), Y. Zhang (Harvard), and the Caenorhabditis Genetics Center, which is funded by NIH Office of Research Infrastructure Programs (P40 0D010440), for worm strains; S. Chalasani (Scripps) for technical advice regarding microfluidics setup; A. Takeishi (Brandeis) for advice about analyzing CMK-1 subcellular localization; Z. Liao, V. Lauper, and L. Schild for superb technical support; J. Kubanek for help with worm tracking; and L. Booth, B. Dulken, S. Han, C.-K. Hu, K. Papsdorf, and P. Singh for critical reading of the paper.

The authors declare no competing financial interests.

*J.P.L. and H.F. contributed equally to this work.

Correspondence should be addressed to either Dr. Miriam B. Goodman or Anne Brunet, Neurosciences Graduate Program, Department of Molecular and Cellular Physiology, Stanford University, B-111 Beckman Center, 279 Campus Drive, Stanford, CA 94305, E-mail: mbgoodman@stanford.edu or abrunet1@stanford.edu. rich in essential resources like food and potential mates, and to avoid barren or perilous ones. Commensurate with its importance, there have been widespread efforts to identify the genes and neural circuits involved in behavioral plasticity. The nematode Caenorhabditis elegans exhibits behavioral plasticity in response to a variety of external stimuli, including temperature, touch, smell, and taste (Ardiel and Rankin, 2010). For this reason and because of its well-characterized nervous system and ease of genetic manipulation, C. elegans is an informative model system for investigating behavioral plasticity.

All animals require salt (sodium chloride) for survival and, as a result, salt-seeking is a highly conserved behavior (Geerling and Loewy, 2008; Hurley and Johnson, 2015). However, high salt can also be a threat especially to small, terrestrial animals like C. elegans because it generates osmotic stress (Choe, 2013; Kim and Jin, 2015). Thus, the worm experiences an intense pressure to balance 
Table 1. Pathways implicated in salt-aversive learning

\begin{tabular}{ll}
\hline Pathway & Citations \\
\hline G-protein signaling & $\begin{array}{l}\text { (Jansen et al., 2002; Hukema et al., 2006; Matsuki et al., 2006; } \\
\text { Sakashita et al., 2008; Adachi et al., 2010; Kunitomo et al., } \\
\text { 2013) } \\
\text { Insulin signaling }\end{array}$ \\
$\begin{array}{l}\text { (Tomioka et al., 2006; Vellai et al., 2006; Adachi et al., 2010; 0da } \\
\text { et al., 2011; Kunitomo et al., 2013) } \\
\text { Synaptic proteins }\end{array}$ & $\begin{array}{l}\text { (Ishihara et al., 2002; Ikeda et al., 2008; Stetak et al., 2009; } \\
\text { Iwata et al., 2011) }\end{array}$ \\
Neurotransmitters & (Hukema et al., 2008; Kano et al., 2008; Stetak et al., 2009) \\
Other & (Saeki et al., 2001; lino and Yoshida, 2009; Sakai et al., 2013) \\
\hline
\end{tabular}

the need for salt with the need to avoid toxic concentrations. A considerable body of evidence establishes that C. elegans nematodes seek salt by performing positive chemotaxis (Ward, 1973; Bargmann and Horvitz, 1991) and this attraction can be transformed into aversion (negative chemotaxis) following conditioning in solutions rich in salt and poor in bacterial food (Saeki et al., 2001; Tomioka et al., 2006). Although several signaling pathways (Table 1), including those linked to insulin signaling (Tomioka et al., 2006; Vellai et al., 2006; Adachi et al., 2010; Oda et al., 2011; Kunitomo et al., 2013), modulate this salt-aversive learning behavior, the majority of mutants analyzed thus far exhibit modest deficits in learning ability. Thus, genes essential for saltaversive learning behavior and their cellular locus of action remain under-studied.

Calcium and calmodulin (CaM)-dependent kinases play evolutionarily conserved roles in the nervous system in signal transduction, synaptic development, and behavioral plasticity. Among other functions, CaM kinases respond to changes in cytosolic calcium levels and regulate gene transcription in the nucleus. Although CaMKII is the most intensively studied, CaMKI and CaMKIV have been found to be important for specific functions such as developmental and activity-dependent growth (Hook and Means, 2001; Wayman et al., 2008; Cohen et al., 2015). Recently, the sole worm ortholog of mammalian CaMKI/IV, CMK-1, has emerged as a versatile regulator of neuronal plasticity in C. elegans. CMK-1 is expressed broadly throughout the worm's nervous system (Kimura et al., 2002; Satterlee et al., 2004), and regulates behavioral plasticity in responses to thermal stimuli (Satterlee et al., 2004; Schild et al., 2014; Yu et al., 2014; Kobayashi et al., 2016). It also regulates developmental plasticity by affecting entry into the "dauer" diapause state in response to food deprivation (Neal et al., 2015), and synaptic plasticity by regulating expression of an AMPA-type glutamate receptor (Moss et al., 2016). However, whether CMK-1 is implicated in salt attraction or its transformation to salt aversion following conditioning is unknown.

Here, we show that CMK-1 is absolutely required for saltaversive learning behavior yet dispensable for salt attraction. In contrast, disrupting the only other CaM kinase encoded by the $C$. elegans genome, UNC-43 CaMKII, or other kinases such as AMP kinase and protein kinase $\mathrm{C}$, has no detectable effect on either positive chemotaxis or its transformation to negative chemotaxis following conditioning in high-salt and the absence of bacterial food. By expressing wild-type CMK-1 in a neuron-specific manner, we find that $c m k-1$ functions in the ASEL and ASER chemosensory neurons known to be required for salt chemotaxis (Bargmann and Horvitz, 1991). Having identified the ASE neurons as a critical cellular locus for $c m k-1$ action, we analyze salt-evoked calcium signals in wild-type and $c m k-1$ mutant ASE neurons. Consistent with prior studies, we show that conditioning sufficient to induce salt-aversive learning blunts salt-evoked calcium signals in ASE cell bodies. Loss of $c m k-1$ has subtle effects on salt-evoked calcium responses in ASE dendrites and axons, affecting ASEL to a greater extent than ASER. Collectively, our results expand the forms of behavioral plasticity that depend upon CMK-1 function in primary sensory neurons.

\section{Materials and Methods}

Worm strains and husbandry. C. elegans strains were cultured using standard conditions at $20^{\circ} \mathrm{C}$ on NGM plates seeded with OP50-1 bacteria. All experiments were performed using adult hermaphrodites. Transgenic worms were generated using microinjection, resulting in formation of extra-chromosomal arrays. A subset of the C. elegans strains we used were generated specifically for this study, the remainder were obtained from our in-house collections, as gifts from colleagues, or from the Caenorhabditis Genetics Center. Table 2 lists all strains, their RRIDs (if available) or origin, and the figures showing data from each strain.

Transgenic strains expressing full-length wild-type CMK-1 in one or both ASE neurons were created for this study using standard transgenesis methods (Evans, 2006). Rescue plasmids encoding wild-type CMK-1 were generated with the three-fragment Multisite Gateway system (Invitrogen), as previously described (Schild et al., 2014). A 2027bp gcy-5 promoter fragment, a $710 \mathrm{bp} g c y-6$ promoter fragment, and a $2682 \mathrm{bp}$ flp-6 promoter fragment were amplified by PCR and cloned into pDONR P4P1r through BP recombination reactions to produce slotl Entry clones. The primers for amplification of the $g c y-5, g c y-6$, and $f l p-6$ promoter fragments were as follows:

gcy-5pF, ggggacaactttgtatagaaaagttgGCTCGTTAACTCTTCACATG; gcy-5pR, ggggactgcttttttgtacaaacttgTTTTCATCAGAATAAGTAATT TTTCGAAAAC;

gcy-6pF, ggggacaactttgtatagaaaagttgACGTAATTGTGTCATTTTTGA AGTC;

gcy-6pR, ggggactgcttttttgtacaaacttgTCACTGGAAAGGAGTTATGAA AATG)

flp-6pF, ggggacaactttgtatagaaaagttgTCGCTTGACTTCTGATG

flp-6pR, ggggactgcttttttgtacaaacttgTTCTGGAATAATCATATTG

For the $g c y-5$ and $g c y$ - 6 strains, expression clones were made through LR reactions between each slot 1 Entry clone, a slot 2 Entry clone containing $c m k-1$ coding sequence (mg271), a slot3 Entry clone containing SL2::mCherry (mg277) and a pDEST R4R3 destination vector. The resulting expression plasmids, dg616[gcy-5p::cmk-1::SL2::mCherry] and dg617 [gcy-6p::cmk-1::SL2:::mCherry], were microinjected together with a [coel::GFP] marker (Addgene \#8937, gift from Piali Sengupta; Miyabayashi et al., 1999) in the gonad of DAG142 cmk-1(ok287) animals according to standard procedures (Evans et al., 2006). The final concentration of each plasmid was $20 \mathrm{ng} / \mu$ l. Stable lines (Table 2) were recovered and epifluorescence microscopy was used to confirm the expression of the trans-spliced mCherry reporter in the expected ASE neurons. The extrachromosomal array in one of the dual rescue lines (DAG721) spontaneously integrated into the genome.

For the $f l p-6$ strains, expression clones were made through LR reactions between each slot1 Entry clone, a slot2 Entry clone containing cmk-1 coding sequence (mg273), a slot3 Entry clone containing GFP:: unc-54 (mg208), and a pDEST R4R3 destination vector. The resulting expression plasmid, pJPL3 [flp-6p::cmk-1::GFP], was microinjected together with a [myo-3p::mCherry] marker (Addgene \#19328) in the gonad of VC220 cmk-1 (ok287) animals according to standard procedures (Evans et al., 2006). The final concentration of the plasmid was $50 \mathrm{ng} / \mu \mathrm{l}$. Stable lines (Table 2) were recovered and epifluorescence microscopy was used to confirm the expression of the GFP reporter in the expected ASE neurons.

Salt conditioning. Following a previously reported method (Tomioka et al., 2006), we transferred adult hermaphrodites from culture plates to $1.5 \mathrm{ml}$ microcentrifuge tubes containing one of two conditioning buffers before each assay. The first buffer contained no added $\mathrm{NaCl}(0 \mathrm{~mm})$ and the second buffer contained $20 \mathrm{~mm} \mathrm{NaCl}$. Both buffers also contained the following (in mM): $5 \mathrm{KPO}_{4}$, $\mathrm{pH} 6.0,1 \mathrm{MgSO}_{4}$, and $1 \mathrm{CaCl}_{2}$. We removed residual bacteria by washing animals once in fresh buffer and conditioned worms for the indicated time periods (between $5 \mathrm{~min}$ and $10 \mathrm{~h}$ ). 
Table 2. List of $C$. elegans strains used in this study

\begin{tabular}{|c|c|c|c|}
\hline Strain name & Genotype & RRID or source & Figure \\
\hline N2 & Wild-type, Bristol & WB-STRAIN:N2_(ancestral) & Throughout \\
\hline RB754 & aak-2(ok524)X & WB-STRAIN:RB754 & Fig. $1 C$ \\
\hline RB781 & pkc-1(0k563) V & WB-STRAIN:RB781 & Fig. $1 C$ \\
\hline VC1052 & unc-43(gk452) IV & WB-STRAIN:VC1052 & Fig. $1 C$ \\
\hline JT200 & unc-43(sa200) IV & WB-STRAIN:JT200 & Fig. $1 C$ \\
\hline VC220 & $c m k-1(0 k 287) I V$ & WB-STRAIN:VC220 & Throughout \\
\hline YT17 & crh-1(tz2) III & WB-STRAIN:YT17 & Fig. $2 D$ \\
\hline MT9973 & crh-1(n3315) III & (Kauffman et al., 2010) & Fig. $2 D$ \\
\hline PS3551 & hsf-1(sy441)I & WB-STRAIN:PS3551 & Fig. $2 D$ \\
\hline GN190 & $\mathrm{cmk}-1(\mathrm{pg} 58) \mathrm{IV}$ & (Schild et al., 2014) & Fig. $4 D$ \\
\hline GN634 & cmk-1(ok287) IV; oyls17[gcy-8p::GFP] V; pgEx167[cmk-1p:::mk-1(WT)::GFP; myo-3p:::mCherry] & This study & Figs. $1 D, 4 A$ \\
\hline DAG307 & cmk-1(ok287) IV; domEx307[cmk-1p:::cmk-1(1-348)T179A; unc-122p::RFP] & (Schild et al., 2014) & Fig. $2 B$ \\
\hline PY8395 & cmk-1(oy21) IV; Ex[ceh-36Dp:::mk-1(WT); unc-122p::dsRed] line 1 & (Neal et al., 2015) & Fig. $3 B$ \\
\hline DAG176 & cmk-1(ok287) IV; domEx176[cmk-1p::cmk-1(WT)::NES:::GFP; unc-122p:::RFP] & (Schild et al., 2014) & Fig. $4 B, C$ \\
\hline DAG178 & cmk-1(ok287) IV; domEx178[cmk-1p:::cmk-1(WT)::GFP:::NLS; unc-122p:::RFP] & (Schild et al., 2014) & Fig. $4 B, C$ \\
\hline ZC1600 & yxEx738[flp-6p::GCaMP3; unc-122p::GFP] & (Luo et al., 2014) & Figs. $5,6 A, B, E, 7 A, C, 7-1 A$ \\
\hline GN637, GN670 & cmk-1(ok287) IV; yxEx738 [flp-6p::GCaMP3; unc-122p:::GFP] & This study & Figs. $6 C-E, 7 B, C, 7-1 B$ \\
\hline DAG142 & cmk-1(ok287) IV & This study & Fig. $3 C$ \\
\hline DAG720 & cmk-1(ok287) IV; domEx720[gcy-5p::cmk-1::SL2::mCherry; gcy-6p::cmk-1::SL2::mCherry; coel::GFP] & This study & Fig. 3-1 \\
\hline DAG721 & cmk-1(ok287) IV; domls721[ [gcy-5p:::cmk-1::SL2:::mCherry; gcy-6p:::cmk-1::SL2:::mCherry; coel::GFP] & This study & Fig. $3 C$ \\
\hline DAG716 & cmk-1(ok287) IV; domEx716[gcy-6p::cmk-1::SL2:::mCherry; coel::GFP] & This study & Fig. 3-1 \\
\hline DAG717 & cmk-1(ok287) IV; domEx717[gcy-6p:::cmk-1::SL2::mCherry; coel::GFP] & This study & Fig. $3 C$ \\
\hline DAG712 & cmk-1(ok287) IV; domEx712[gcy-5p:::cmk-1::SL2::m Cherry; coel:::GFP] & This study & Fig. 3-1 \\
\hline DAG713 & cmk-1(ok287) IV; domEx713[gcy-5p:::cmk-1::SL2::mCherry; coel::GFP] & This study & Fig. $3 C$ \\
\hline
\end{tabular}

Chemotaxis assays. We adapted methods for assessing salt chemotaxis and salt-aversive learning from Tomioka et al. (2006) and diagram the process in Figure $1 \mathrm{~A}$. We generated radial salt gradients on assay plates by placing a 5-mm-thick high-salt agar plug $5 \mathrm{~mm}$ away from the edge of the $3.5 \mathrm{~cm}$ assay plate (with a 5-mm-thick agar plate) and leaving it in place for $3 \mathrm{~h}$ at $23^{\circ} \mathrm{C}$. The high-salt $2 \%$ agar plug contained the following (in $\mathrm{mm}$ ): $5 \mathrm{KPO}_{4}, \mathrm{pH} 6.0,1 \mathrm{MgSO}_{4}, 1 \mathrm{CaCl}_{2}$, and $100 \mathrm{NaCl}$. The assay $2 \%$ agar assay plate contained the following (in $\mathrm{mm}$ ): $5 \mathrm{KPO}_{4}, \mathrm{pH} 6.01$, $\mathrm{MgSO}_{4}$, and $1 \mathrm{CaCl}_{2}$. Immediately before the start of the chemotaxis assay, the plug was removed and a drop $(1 \mu \mathrm{l})$ of sodium azide $(0.1 \mathrm{M})$ was spotted on opposite sides of the assay plate to immobilize animals near their final positions.

Conditioned worms were deposited in the middle of a $3.5 \mathrm{~cm}$ diameter radial salt gradient assay plate. Residual buffer was wicked off with a Kimwipe, and worms were allowed to crawl for $30 \mathrm{~min}$ at $20^{\circ} \mathrm{C}$. The number of worms in each region (origin, low salt, high salt) was counted and used to compute a chemotaxis index:

$$
C I=\frac{\left(N_{\text {high salt }}-N_{\text {low salt }}\right)}{\left(N_{\text {total }}-N_{\text {origin }}\right)}
$$

where $C I$ is the chemotaxis and $N$ is the number of worms in the indicated category. For each assay, we collected well-fed worms from a single growth plate $(6 \mathrm{~cm})$ into one $1.5 \mathrm{ml}$ microcentrifuge tube for conditioning and then divided this sample into three equal aliquots to perform chemotaxis assays in triplicate.

Locomotion assay. We collected adult (age: $1 \mathrm{~d}$ ) worms from growth plates and conditioned them for $180 \mathrm{~min}$, as described above for chemotaxis assays. Locomotion speed was assessed in the absence of food on standard NGM plates and we allowed animals to acclimate for $5 \mathrm{~min}$ before imaging. We collected short $(1 \mathrm{~min})$ movies at $6 \times$ magnification at $20 \mathrm{frames} / \mathrm{s}$ (50 ms/frame) with a CCD camera (Mightex, M/N SMEB050-U) mounted on zoom lens (Navitar) with a C-mount adaptor and
$0.5 \times$ lens and an illumination ring positioned in the same plane as the agar plate and providing radial illumination around it. Tracks showing locomotion were generated using the automated Parallel Worm Tracker software (Ramot et al., 2008) and were used to compute the average speed/track. The average speed of each sample was determined by computing the ensemble average of speed/track.

Colocalization imaging. Colabeling imaging for $f l p-6 p$ and $c m k-1 p$ fluorescent reporters was performed on an Axioplan 2 Zeiss epifluorescence microscope equipped with an Axiocam camera and a $40 \times$ objective (air, $\mathrm{NA}=0.95$ ), as previously described (Hostettler et al., 2017).

Subcellular localization imaging. We collected adult (age: $1 \mathrm{~d}$ ) worms from growth plates and conditioned them for $180 \mathrm{~min}$. In buffer containing the following (in mM): $5 \mathrm{KPO}_{4}, \mathrm{pH} 6.0,1 \mathrm{MgSO}_{4}, 1 \mathrm{CaCl}_{2}$, and 0 $\mathrm{NaCl}, 20 \mathrm{NaCl}$, or $0 \mathrm{NaCl}$ with OP50-1 bacteria for $0,10,30$, and $180 \mathrm{~min}$. After conditioning we put the animals into a microfluidic chip (Nekimken et al., 2017) to restrict the worm's motility during imaging. We took images using a Leica DMI 4000 B microscopy system, consisting of a cyan LED (Spectra X light engine, Lumencor), a fluorescence cube with a beam splitter (Chroma, Q495lp) and an emission filter (Chroma, HQ500lp), a $63 \times / 1.32$ NA oil objective (Leica), and a Hamamatsu OrcaFlash 4.0LT digital CMOS camera. The subcellular localization was quantified by comparing the relative average fluorescence intensity of a hand-drawn ROI covering the nucleus versus the average fluorescence intensity of an ROI covering the cytoplasmic compartments each subtracted by the average fluorescence of an ROI outside the cell.

In vivo calcium imaging. We collected worms (age: $1 \mathrm{~d}$ ) expressing the $f l p-6 p:: G C a M P 3$ transgene and conditioned them for at least $180 \mathrm{~min}$, using the same procedures and buffers as described for chemotaxis assays. Following conditioning, worms were maintained in the conditioning buffer and positioned in a polydimethylsiloxane microfluidic device (MicroKosmos) that immobilizes the worm's body while leaving the nose exposed to laminar buffer flow. The chip design is based on a device 
A

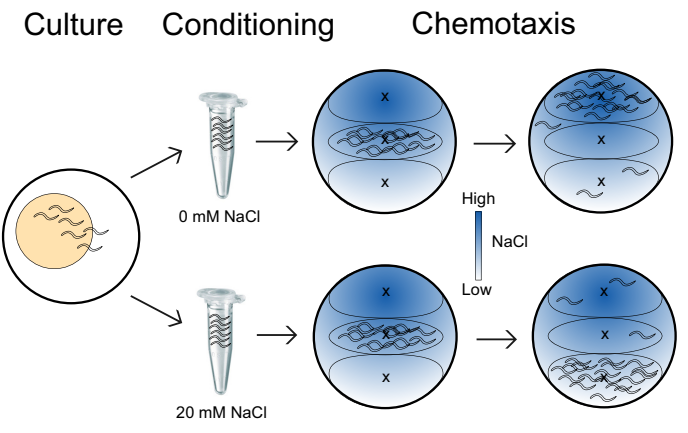

B

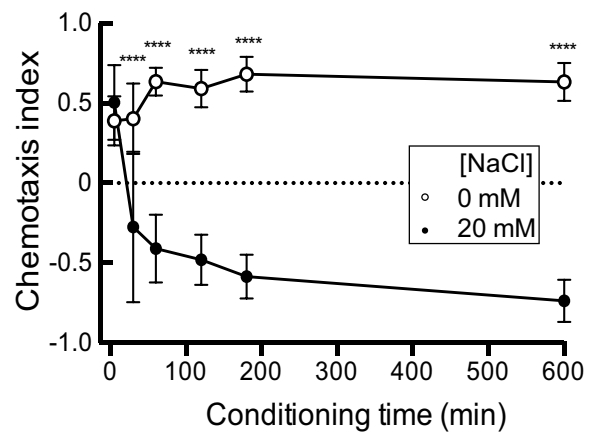

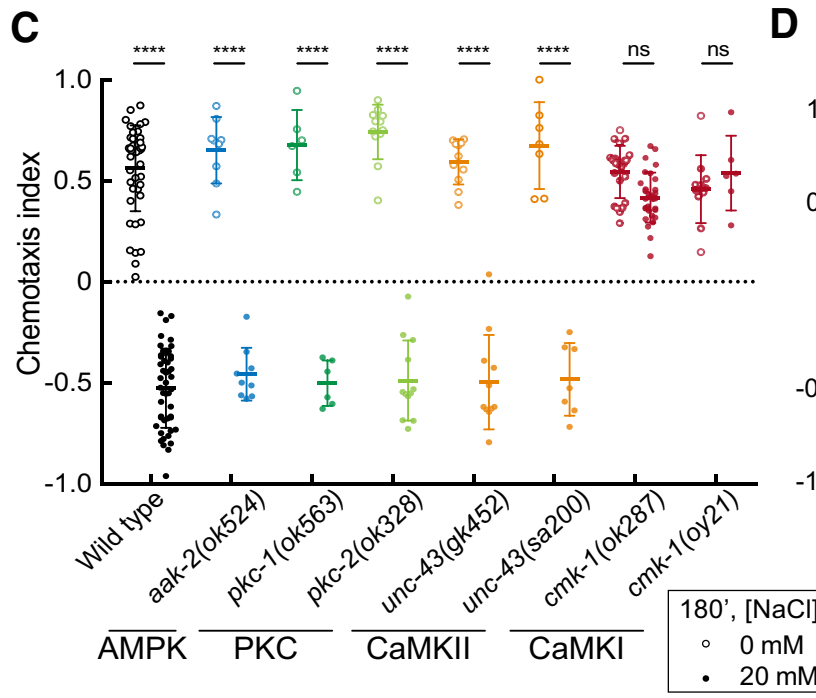

D

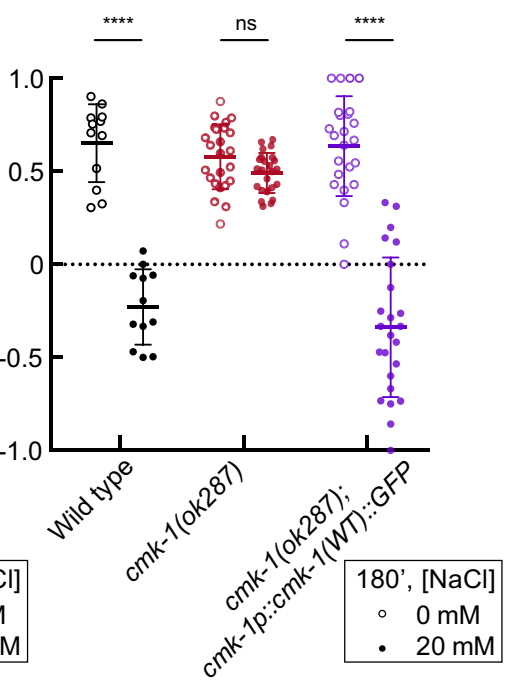

Figure 1. CMK-1 is required for salt chemotaxis learning. $\boldsymbol{A}$, Procedure for testing salt-aversive learning. Left to right: Young adult (Day 1) worms are collected from culture plates, conditioned in food-free buffer containing 0 or $20 \mathrm{~mm} \mathrm{NaCl}$ for $180 \mathrm{~min}$, unless otherwise specified, transferred to the center of an assay plate pretreated to create a radial salt gradient, allowed to migrate for $30 \mathrm{~min}$. Results were quantified by computing a Chemotaxis Index (Cl; see Materials and Methods). B, Salt-aversion development following several hours of conditioning in wild-type (N2) adults. Symbols are the mean $\pm S D$ of three to nine assays for animals conditioned in $0 \mathrm{~mm} \mathrm{NaCl}$ (open circles) or $20 \mathrm{~mm} \mathrm{NaCl}$ (filled circles) for the indicated times. Data were pooled from four independent

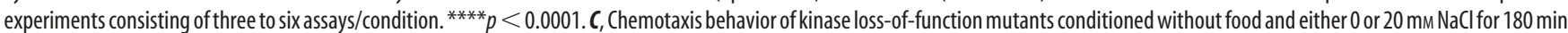
$\left(180^{\prime}\right)$. Each circle is the result of a single assay; horizontal bars indicate the mean \pm SD of all the data. Data were pooled from at least two independent experiments consisting of three to six replicates/condition. All assays were performed blind to genotype. A two-way ANOVA revealed a significant interaction between genotype and condition $(F(7,237)=62.34, p<0.0001)$. D, Transgenic expression of wild-type CMK-1::GFP under its own promoter restores wild-type salt-aversive learning to $c m k-1(0 k 287)$ mutants. Each symbol represents the results of a single assay; horizontal bars indicate mean \pm SD of all assays. Data were pooled from at least two independent experiments consisting of three to six assays/condition. A two-way ANOVA revealed a significant interaction between genotype and condition $(F(2,114)=44.2, p<0.0001)$. Sidak's posthoc tests were used to assess the effect of conditioning in panels $\boldsymbol{C}$ and $\boldsymbol{D}$. The results are indicated by asterisks: ${ }^{*},{ }^{* *}$, and ${ }^{* * * *}$ denote $p<0.1, p<0.01$, and $p<0.0001$ and ns denotes not significant.

reported by Chronis et al. (2007). Under baseline conditions, the worm's nose was exposed to a $\mathrm{NaCl}$-free buffer for $<10$ s before the start of image acquisition. Chemosensory stimulation and imaging occurred during 90 s time blocks consisting of a control period ( $10 \mathrm{~s})$, a $40 \mathrm{~s} \mathrm{NaCl}$ pulse $(20$ $\mathrm{mm})$, and a recovery period (40 s). The solutions were switched as described by Chalasani et al. (2007) by a three-way valve (778360, The Lee Company) that was controlled by a power supply (6212B, Hewlett Packard). We added fluorescein $(\sim 20 \mathrm{~mm})$ to the solution coming from the two side channels. The stream from these two channels is used to redirect the flow of the $\mathrm{NaCl}$-free and the $20 \mathrm{~mm} \mathrm{NaCl}$ buffer and to visualize solution switches.

We performed calcium imaging of ASE neurons of worms held in the chip, which was mounted on a Leica DMI 4000 B equipped for epifluorescence imaging based on a cyan LED light source (Spectra X light engine, Lumencor), image splitting optics (W-view Gemini, Hamamatsu), and an sCMOS camera (Orca-Flash 4.0LT, Hamamatsu). We mounted a longpass filter (Chroma, Q495lp) and an emission filter (Chroma, HQ500lp) in the beam splitter to collect GCaMP3 fluorescence. Fluorescence sig- nals were collected using a $20 \times / 0.4$ NA objective (Leica) and recorded at $10 \mathrm{~Hz}$ for the entire $90 \mathrm{~s}$ of the stimulus protocol. This setup was also used to perform calcium imaging of indentation-evoked calcium responses in C. elegans touch receptors neurons (Nekimken et al., 2017; Fehlauer et al., 2018).

All image sequences were analyzed using custom code running inside Fiji software (Schindelin et al., 2012). Briefly, a desired region of the cell, the sensory ending (dendrite), cell body, or axons was selected manually in the first image of each sequence. In the first image the program identifies the brightest $(b)$ and the dimmest $(d)$ pixel in an area $(5 \times 5 \mu \mathrm{m}$ for cell bodies and $1 \times 1 \mu \mathrm{m}$ for dendrites and axons) around this selection. To track the cell region in the following images the brightest pixel of the previous image is used as the midpoint to search for the new brightest and dimmest pixel as described before. Based on the fluorescence of the brightest $\left(F_{b}\right)$ and the dimmest $\left(F_{d}\right)$ pixel a threshold background. For background correction, the intensity of the background $\left(F_{b g}\right)$ was subtracted from the intensity of the region of cell $(F)$. The difference was divided by the mean prestimulus intensity of the region of cell $\left(F_{0}\right.$, cor- 
rected by $F_{b g}$ ). The result is the background subtracted, relative change of the intensity of the cell region, which is reported as a percentage: $F / F_{0}=$ $100 \times\left[\left(F-F_{b g}\right) /\left(F_{0}-F_{b g}\right)\right]$.

The signals we report in Figures 5, 6, 7 are normalized to fluorescence levels measured before application of a salt pulse in all conditions and genotypes and recordings were normalized individually before being averaged. Although this normalization strategy differs from those used previously in which stimulus-evoked calcium signals are normalized to values immediately before an up-step or a down-step (Suzuki et al., 2008; Ortiz et al., 2009; Thiele et al., 2009; Oda et al., 2011; Rabinowitch et al., 2014), it enables a simple experimental design in which a single pulse can be used to interrogate responses to both up-steps and down-steps. We also normalized somatic ASER responses to values immediately before a down-step and include the pooled, average responses as insets in Figure $6 B$ and $D$.

The mean traces of the ASEL and the ASER dendrites were fitted individually with functions describing the ON response in ASEL and the OFF response in ASER as the difference of two exponential functions and the OFF response in ASEL and the ON response in ASER as an exponential function.

$$
\begin{gathered}
d_{A S E L}(t)=\left\{\begin{array}{c}
1: t<11.5 s \\
1-\left(A_{1} \cdot\left(e^{-k_{1} \cdot t}-e^{-k_{2} \cdot t}\right)\right): 11.6 s<t<51.1 s \\
1-\left(\left(A_{2}-1\right) \cdot\left(e^{-k_{3} \cdot t}\right)\right): 51.2 s<t,
\end{array}\right. \\
d_{A S E R}(t)=\left\{\begin{array}{c}
1: t<11.5 s \\
1-A_{1}+A_{1} \cdot\left(e^{-k_{1} \cdot t}\right): 11.6 s<t<51.1 s \\
A_{1}+\left(A_{2}\left(e^{-k_{2} \cdot t}-e^{-k_{3} \cdot t}\right)\right): 51.2 s<t .
\end{array}\right.
\end{gathered}
$$

Experimental design and statistical analyses. This study used adult C. elegans hermaphrodites (age: day 1, first day of egg-laying) for all experiments, which included chemotaxis assays, analysis of gene and protein expression by fluorescence microscopy, and neuronal activity by imaging a genetically-encoded calcium indicator. For each chemotaxis assay, we placed between 30 and 450 worms on an assay plate and analyzed at least three assay plates for each condition. We pooled data from at least two independent replicates, which were performed blind to genotype, except in assays involving strains carrying transgenic arrays. In the latter case, genotype was determined post hoc by visual inspection for the presence or absence of the transgene and behavioral performance was scored for both genotypes in parallel. To achieve this goal, we used sodium azide $(0.1 \mathrm{M})$ to immobilize animals after the $30 \mathrm{~min}$ chemotaxis assay period and counted animals of both genotypes on the stage of stereomicroscope equipped for epifluorescence.

We built and analyzed four independent transgenic lines coexpressing CMK-1::GFP from a flp-6 promoter active in the ASE neurons and mCherry from the cmk-1 promoter (DAG610, DAG611, DAG612, DAG613). We analyzed the subcellular position of CMK-1 using a transgenic line expressing CMK-1::GFP from a flp-6 promoter (10-18 neurons; see Fig. $4 A)$ and verified subcellular localization of strains where CMK-1 was targeted to the nucleus or cytoplasm (14-37 neurons; see Fig. 4C). We analyzed salt-evoked calcium transients in animals held in a microfluidic trap. In transgenic animals with the $y x E x 738$ [flp-6p::GCaMP3 + unc-122p:: GFP] in both ASE neurons, we analyzed signals in 10-27 individual ASER and ASEL neurons across conditions and genotypes. Except in rare instances (4-6 animals), we analyzed a single ASE neuron in each animal. Animals expressing $y x E x 738$ [flp-6p::GCaMP3 + unc-122p::GFP] in either ASER or ASEL, but not both, were rare. As a result, we analyzed signals in four ASEL neurons and six ASER neurons. These data were collected during three independent imaging sessions. We generated two strains expressing $y k E x 738$ in a cmk-1(ok287)-null mutant background, GN637 and GN670, and pooled the results after determining that calcium signals were indistinguishable between the two strains.

Data were tested for normality using the D'Agostino and Pearson omnibus normality test. Data passing this test were compared for differences using two-way ANOVA followed by Sidak's post hoc test. These analyses were performed using Prism 6/7 (GraphPad). Data that did not pass normality were compared using the Wilcoxon rank sum test and adjusted for multiple hypothesis testing using the Benjamini-Hochberg method using R Studio. The outcomes of these statistical analyses are given in each figure legend.

\section{Results}

As a first step in our investigation of salt-aversive learning in $C$. elegans, we determined the relationship between conditioning time and the conversion of positive salt chemotaxis (attraction) to negative salt chemotaxis (aversion). We conditioned animals in buffer with or without salt in the absence of bacterial food and tested their ability to perform chemotaxis (Fig. 1A). Under initial conditions, the chemotaxis index was close to +1 , which would indicate perfect attraction (see Materials and Methods). As shown in Figure $1 B$, animals converted attraction to aversion over a few hours, and the conversion followed an approximately exponential time course. This time course was fit by a single exponential function (time constant, $\tau=21.7 \mathrm{~min}$ ). Control animals, conditioned without salt and in the absence of bacterial food, continued to perform positive chemotaxis even after $10 \mathrm{~h}$ of conditioning. This analysis reveals that conditioning animals for $180 \mathrm{~min}(3 \mathrm{~h})$ was sufficient to saturate the aversion response. Thus, we conditioned animals for $180 \mathrm{~min}$ in the absence of bacterial food without salt (0 mM) or with $20 \mathrm{~mm}$ salt for all subsequent experiments, unless otherwise specified.

\section{CMK-1 is essential for salt-learning behavior in C. elegans}

Protein kinases often transduce extracellular signals into cellular responses. To understand whether and how kinases contribute to salt-aversive learning, we compared the learning ability of wildtype worms to that of worms carrying mutations in several protein kinases known to be involved in relaying key environmental stimuli: AMP kinase (AMPK), protein kinase C (PKC), and CaM kinases (CaMKI and II). Loss-of-function mutants in the worm AMPK [aak-2(ok524)], PKC [pkc-1(ok563), pkc-2(ok328)], and CaMKII genes [unc-43(gk452) and unc-43(sa200)], behaved like wild-type worms, suggesting that these genes are dispensable for both salt seeking and for salt-aversive learning (Fig. 1C). By contrast, $c m k-1$ loss-of-function mutants, $c m k-1(o k 287)$ and cmk-1(oy21), performed positive chemotaxis regardless of whether they were conditioned with or without salt. The defect in salt-aversive learning is due to the loss of $c m k-1$ function because re-expressing wild-type CMK-1 protein under its endogenous promoter was sufficient to restore salt-aversive learning to cmk-1(ok287) mutants (Fig. 1D). The defect does not appear to be due to a change in the conditioning time required for learning, however, as $c m k-1$ mutants failed to convert salt attraction into aversion even after $10 \mathrm{~h}$ of conditioning. Indeed, the chemotaxis index of $\mathrm{cmk}-1$ worms conditioned in the presence and absence of $20 \mathrm{~mm} \mathrm{NaCl}$ for $10 \mathrm{~h}$ was $0.59 \pm 0.21$ (mean $\pm \mathrm{SD}, n=6$ ) and $0.51 \pm 0.07$ (mean $\pm \mathrm{SD}, n=6$ ), respectively. The failure of $c m k-1$ mutants to convert salt attraction into salt avoidance was not due to general defects in salt-sensing or in locomotion since naive $c m k-1$ mutants performed like wild-type animals (Fig. $1 B$ ) and had average locomotion speeds similar to wild-type when assayed on a sterile agar surface: $90 \pm 48 \mu \mathrm{m} / \mathrm{s}$ (mean $\pm \mathrm{SD}, n=$ 37 ) and $115 \pm 55 \mu \mathrm{m} / \mathrm{s}$ (mean $\pm \mathrm{SD}, n=37$ ) for $c m k-1$ and wild-type, respectively. These speeds fall within the range of previously reported values (Ramot et al., 2008). Collectively, these results indicate that CaMKI, but not CaMKII or other kinases tested in this study, is essential for salt-aversive learning but not for salt chemotaxis per se. 
A

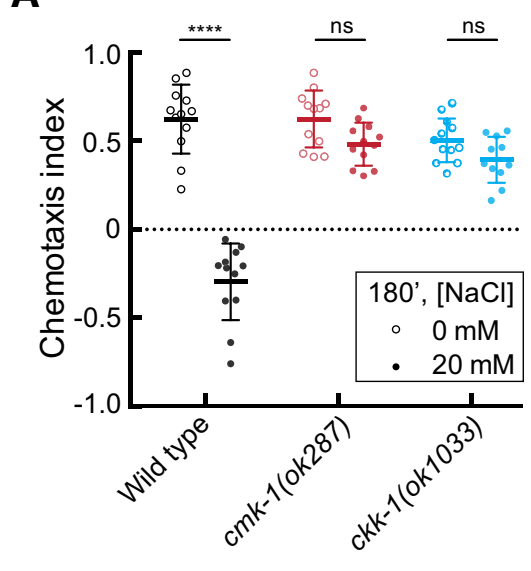

C

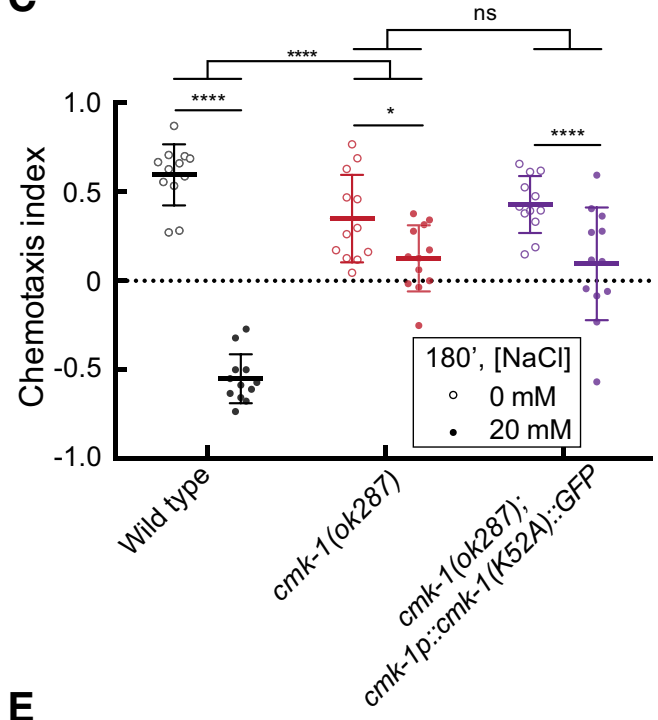

B
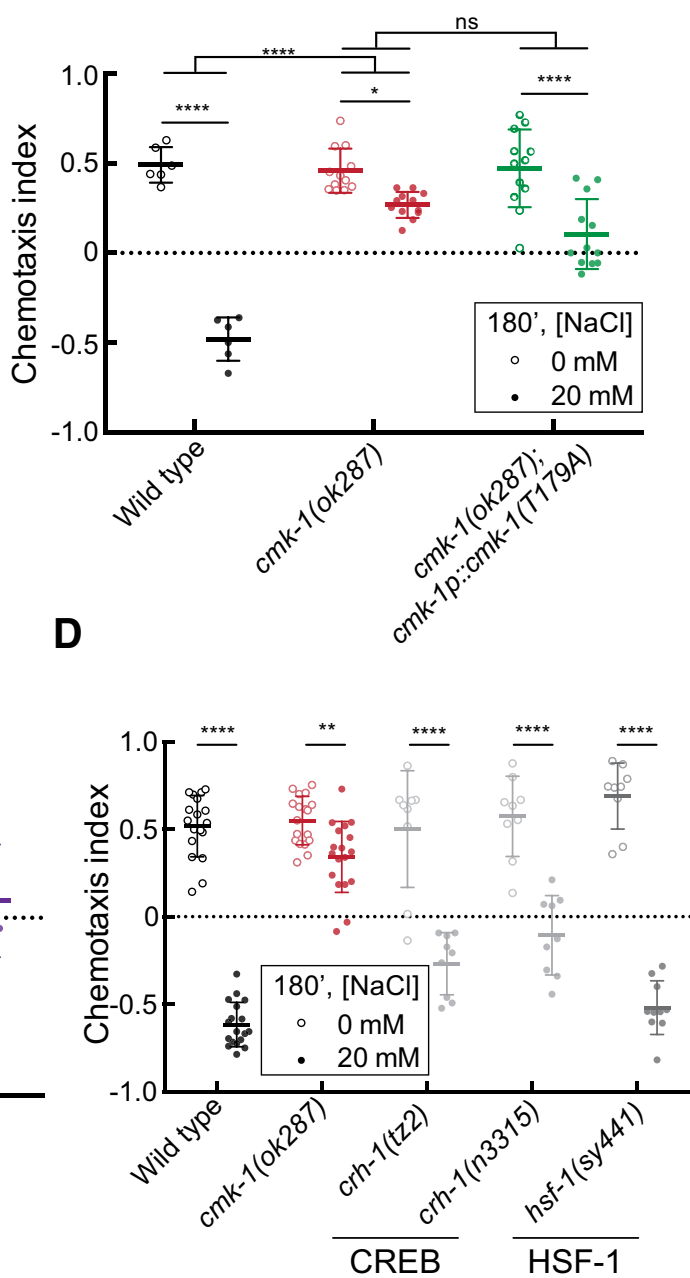

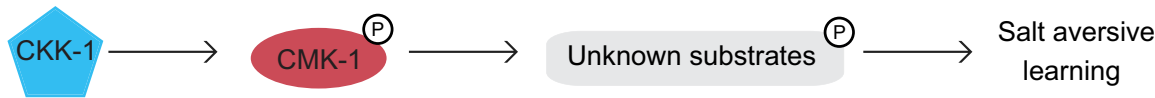

Figure 2. Salt-aversive learning requires CaMKK, CKK-1, and CMK-1 kinase activity. $\boldsymbol{A}$, CKK-1 is needed for salt-aversive learning. A two-way ANOVA revealed a significant interaction between genotype and condition $(F(2,65)=47.37, p<0.0001)$ and Sidak's post hoc test was used to test the effect of conditioning. $\boldsymbol{B}$, A CMK-1 mutant that cannot be phosphorylated fails to restore salt-aversive learning to $\mathrm{cmk}$ - 1 mutants. A two-way ANOVA across all genotypes and conditions revealed a significant interaction between genotype and condition $(F(2,54)=26.17, p<0.0001)$ and Sidak's post hoc test revealed a significant effect of conditioning for all genotypes. A two-way ANOVA between wild-type and $\mathrm{cmk}$ - 1 ( $0 \mathrm{k} 287$ ) revealed a significant interaction between genotype and condition $(F(1,32)=112, p<0.0001)$ and a two-way ANOVA between cmk-1(ok287) and cmk-1(ok287);cmk-1p::cmk-1(T179A) revealed a nonsignificant interaction between genotype and condition $(F(1,44)=3.521, p=0.0672)$. C, A kinase-defective CMK-1(K52A) mutant protein cannot restore salt-aversive learning to cmk-1(ok287) mutants. A two-way AN0VA across all genotypes and conditions revealed a significant interaction between genotype and condition $(F(2,66)=33.96, p<0.0001)$ and a Sidak's post hoc test revealed a significant effect of conditioning for all genotypes. A two-way ANOVA between wild-type and cmk-1(ok287) revealed a significant interaction between genotype and condition $(F(1,44)=71.16, p<0.0001)$ and a two-way AN0VA between $\mathrm{cmk-1}(\mathrm{ok287})$ and $\mathrm{cmk}$-1(ok287);cmk-1p::cmk-1(K52A)::GFP revealed a nonsignificant interaction between genotype and condition $(F(1,44)=0.6384, p=0.4286) . D$, Behavior of known CMK-1 substrates. A two-way ANOVA revealed a significant interaction between genotype and condition $(F(4,117)=34.05, p<0.0001)$. Sidak's post hoc test was used to assess the effect of conditioning for each genotype. For each panel in this figure, data were pooled from at least two independent experiments, consisting of three to six assays/condition performed blind to genotype. Animals of the indicated genotypes were conditioned for $180 \mathrm{~min}$ in either 0 or $20 \mathrm{~mm} \mathrm{NaCl}$ and tested in a salt chemotaxis assay. Each symbol represents the results of a single assay; open and closed symbols indicate animals conditioned in 0 and $20 \mathrm{~mm} \mathrm{NaCl}$, respectively, and bars indicate mean \pm SD of all assays conducted under the indicated condition and genotype. $\boldsymbol{E}$, A schematic model, where CMK-1 is activated via phosphorylation by its upstream kinase CKK-1, and subsequently activates unknown downstream substrates to regulate salt-aversive learning. Sidak's posthoc tests were used to assess the effect of conditioning in panels $\boldsymbol{A}-\boldsymbol{D}$. The results are indicated by asterisks: ${ }^{*}$, ${ }^{*}$, and ${ }^{* * *}$ denote $p<0.1, p<0.01$, and $p<0.0001$ and ns denotes not significant.

\section{Activation of CaMKI by its upstream kinase and CaMKI} kinase activity are necessary for salt-aversive learning CMK-1 is a substrate of CaM kinase kinase (CaMKK; CKK-1 in worms) via phosphorylation of threonine 179 (Eto et al., 1999; Wayman et al., 2008) and $c k k-1$ is coexpressed with $c m k-1$ in many neurons (Kimura et al., 2002). To determine whether or not phosphorylation of CMK-1 was needed for salt-aversive learning, we analyzed $c k k-1$ mutants and found that these mu- tants also exhibit defects in salt-aversive learning (Fig. 2A). We then tested the learning behavior of $c m k-1$ (ok287) mutants overexpressing a form of CMK-1 harboring a mutation ablating the CKK-1 phosphorylation site (T179A; Schild et al., 2014). As expected if phosphorylation of CMK-1 by CKK-1 were critical for salt-aversive learning, transgenic animals expressing CMK-1(T179A) were unable to convert salt attraction into aversion (Fig. $2 B$ ). These results are consistent with a model in which $c k k-1$ regulates 
A
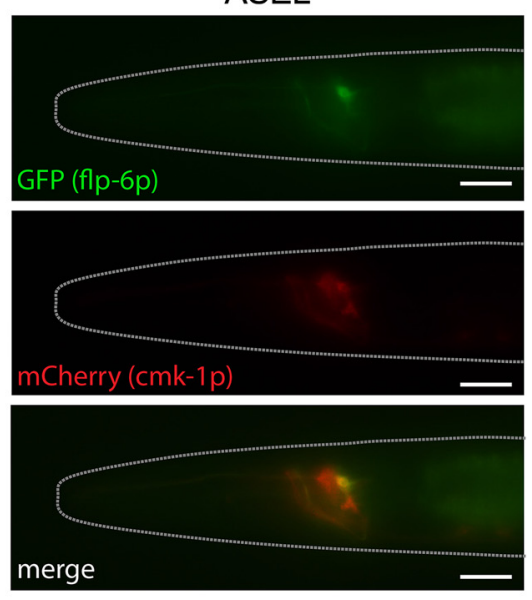

ns

B

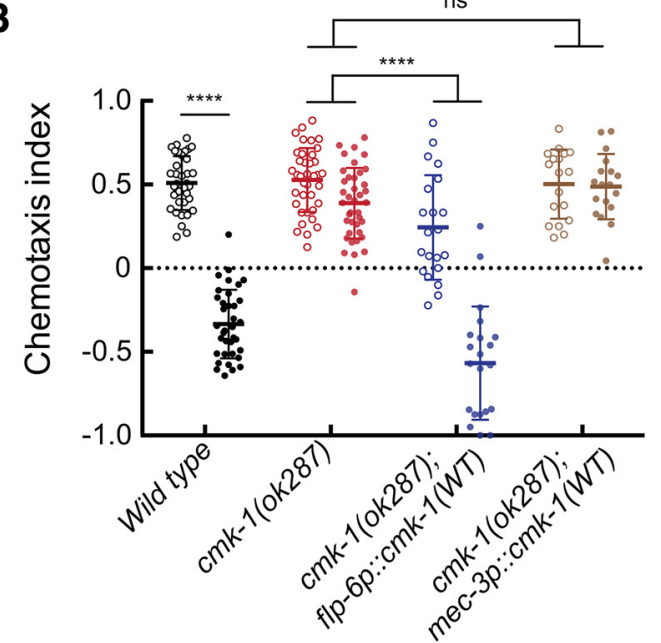

C

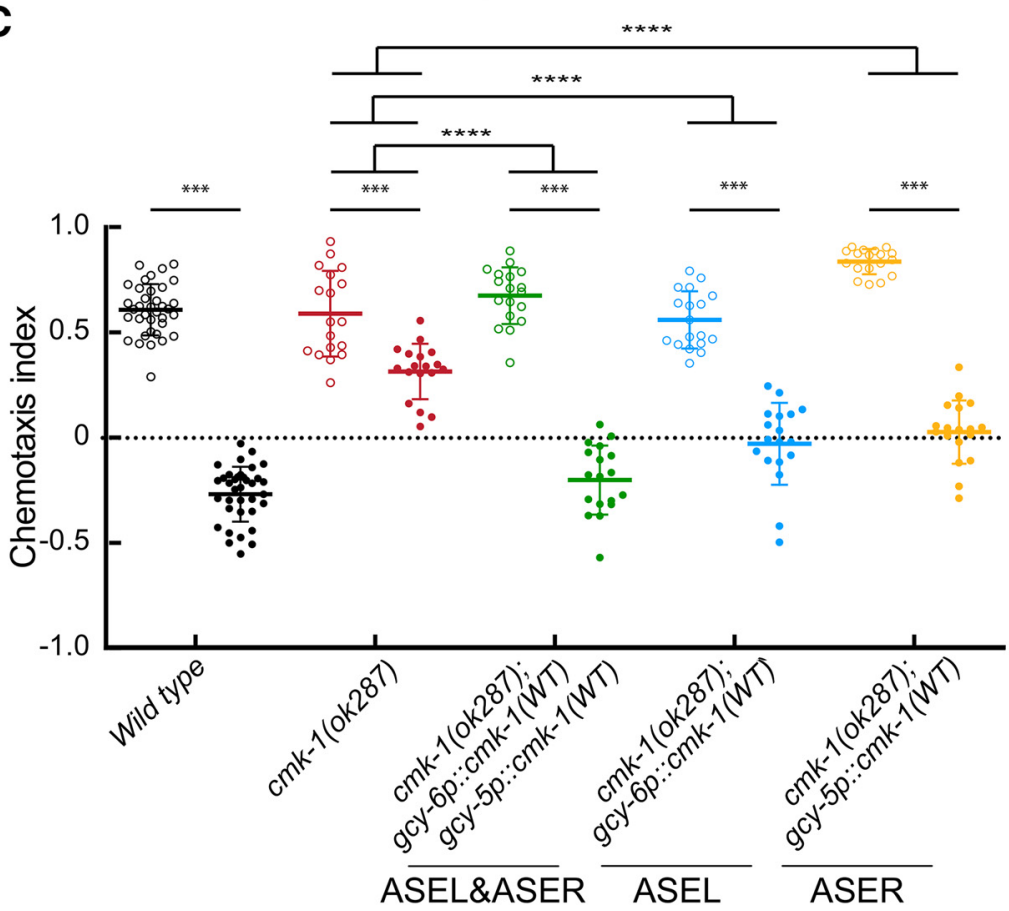

Figure 3. CMK-1 acts in ASE neurons to regulate learning. A, CMK-1 is expressed in ASE sensory neurons. GFP expressed under the flp-6 promoter (known to drive expression in ASE neurons) colocalizes with mCherry expressed under the $\mathrm{cmk}$ - 1 promoter in salt-aversive learning upstream of $c m k-1$ through phosphorylation of the CMK-1 protein, but do not exclude alternative models in which CKK-1 and CMK-1 act in separate pathways.

To determine whether the kinase activity of CMK-1 is needed for salt-aversive learning, we tested a strain overexpressing CMK-1(K52A), a mutant isoform that cannot function as a kinase, but lacking endogenous CMK-1 (Schild et al., 2014). We found that transgenic worms expressing the kinase-dead CMK-1 isoform had intact salt attraction behavior, but had defects in salt-aversive learning like $c m k-1$ (ok287) mutants (Fig. 2C). This result suggests that CMK-1 regulates salt-aversive learning behavior through its function as a kinase.

$\leftarrow$

both ASE neurons of wild-type worms. Shown are the ASEL (left) and ASER (right) neurons from the same worm imaged using epifluorescence microscopy. Worms are oriented anterior side to the left and dorsal side to top of the image. Scale bar, $20 \mu \mathrm{m}$. Similar results were observed in four (of 4 ) independent transgenic lines. $\boldsymbol{B}$, Behavior of $\mathrm{cmk}-1$ (ok287) (left) and cmk-1(oy21) (right) mutants expressing wild-type CMK-1 under neuron-specific promoters. Each circle represents the results of a single assay and bars indicate mean \pm SD; open and closed circles indicate conditioning in the absence and presence of $20 \mathrm{~mm} \mathrm{NaCl}$, respectively. Data were pooled from two independent experiments, where each experiment consisted of three to six plates per condition. Left, A twoway ANOVA across all genotypes and conditions revealed a significant interaction between genotype and condition $(F(3,220)=51, p<0.0001)$. A two-way ANOVA between cmk-1 (ok287) and cmk-1(ok287);flp-6p:::cmk-1(WT):::GFP revealed a significant interaction between genotype and condition $(F(1,116)=48.67, p<0.0001)$. Similar results were observed in animals carrying a second, independent $\mathrm{cmk}$ 1(ok287); flp-6p:::cmk-1(WT)::GFP transgene, which had an average chemotaxis index of $-0.64 \pm 0.12$ (mean $\pm S D$, $n=6$ ) and $0.28 \pm 0.45$ (mean $\pm S D, n=6$ ) with and without conditioning in $20 \mathrm{~mm} \mathrm{NaCl}$, respectively. A two-way ANOVA between $c m k-1(0 k 287)$ and cmk-1(ok287);mec-3p: cmk-1(WT)::GFP revealed a nonsignificant interaction between genotype and condition $(F(1,110)=2.318, p=$ $0.1308)$. Right, $A$ two-way ANOVA revealed a significant interaction between genotype and condition $(F(2,65)=45.12$, $p<0.0001)$. C, Behavior of cmk-1 (ok287) mutants expressing wild-type CMK-1 under ASEL- and ASER-specific promoters. Each circle represents the results of a single assay and bars indicate mean $\pm S D$; open and closed circles indicate conditioning in the absence and presence of $20 \mathrm{~mm} \mathrm{NaCl}$, respectively. For clarity, data were pooled from three independent experiments with the following strains: N2, DAG142, DAG712, DAG716, and DAG721, where each experiment consisted of 6 plates per condition. A two-way ANOVA across all genotypes and conditions revealed a significant interaction between genotype and condition $(F(4,170)=29.5, p<$ 0.0001). Data for independent ASEL, ASER, and ASEL\&ASER rescue lines are shown in Figure 3-1 (available at https://doi. org/10.1523/JNEUROS(I.1611-17.2018.f3-1). Sidak's posthoc tests were used to assess the effect of conditioning and to compare genotypes in panels $\boldsymbol{B}$ and $\boldsymbol{C}$. The results are indicated by asterisks: ${ }^{* *}$ and ${ }^{* * * *}$ denote $p<0.001$ and $p<0.0001$, respectively and ns denotes not significant. 
A

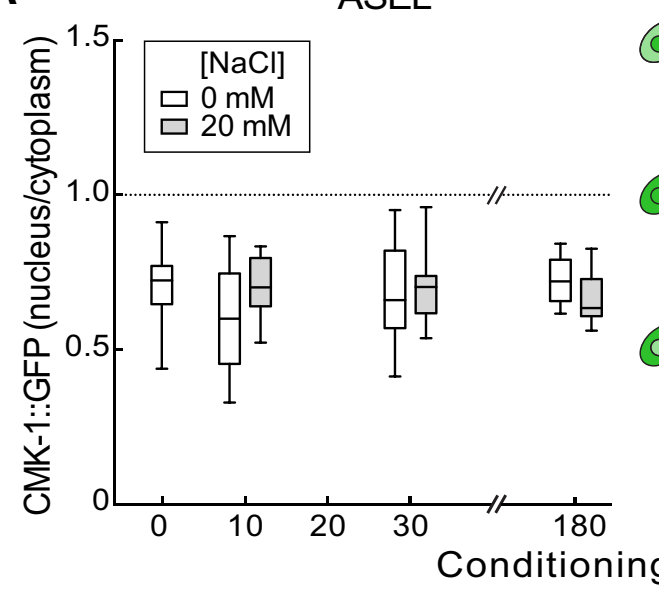

B
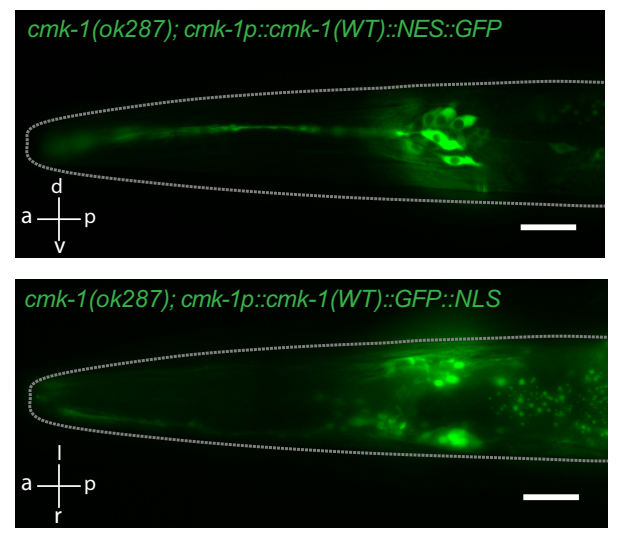

D

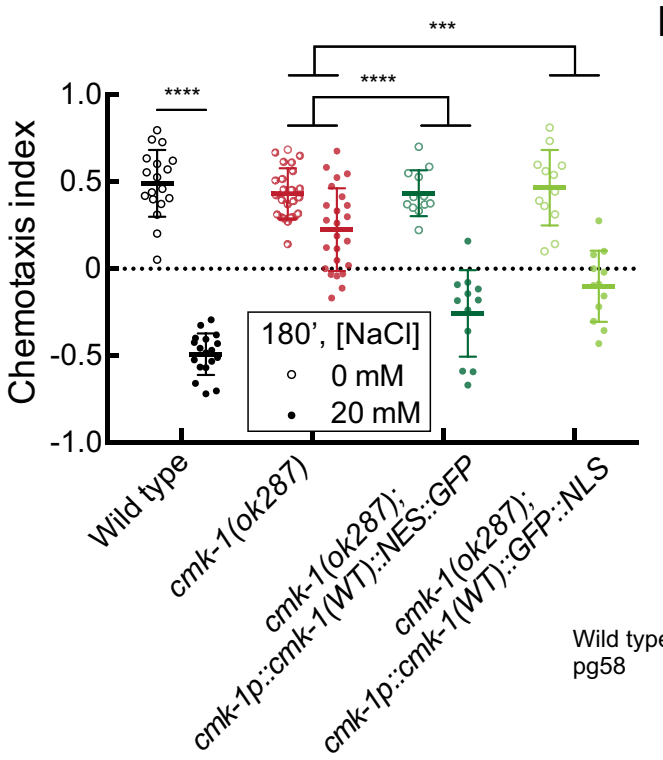

(2)
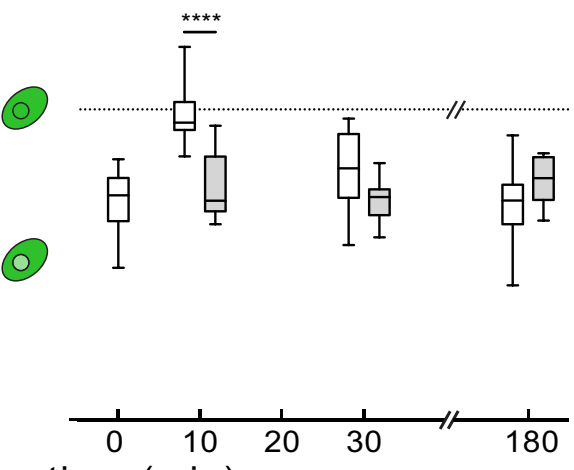

C

E
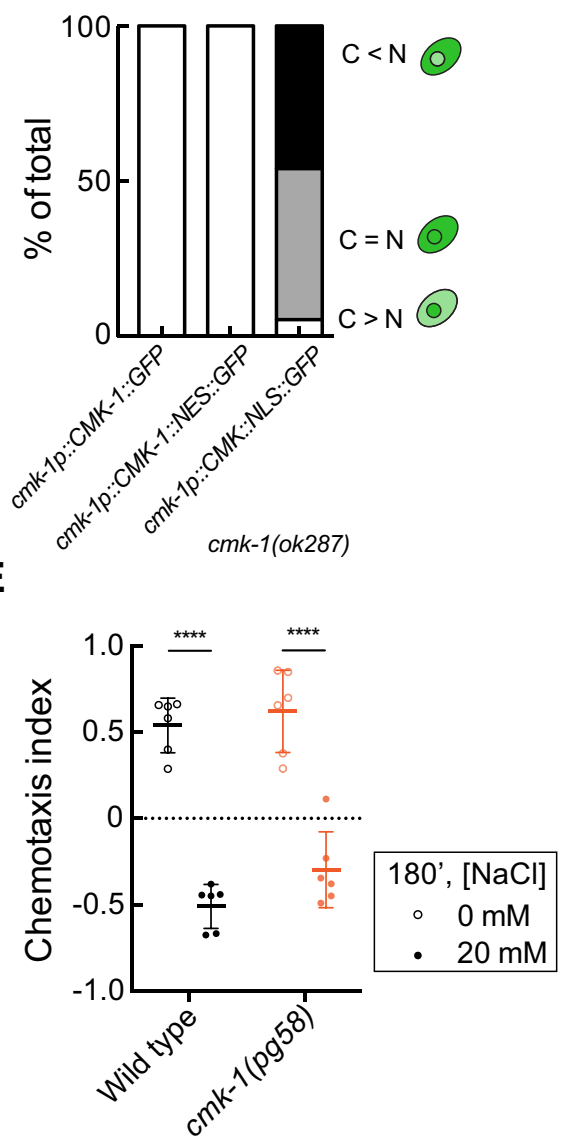

NLS

Kinase domain

Autoinhibitory domain NES

Figure 4. Salt conditioning does not depend on the exact subcellular localization of the CMK-1 protein. $A$, Subcellular localization of CMK-1 in the ASE neurons. The ratio of fluorescence in the nuclear compartment versus the cytoplasmic compartment is plotted separately for ASEL (left) and ASER (right). Each box-and-whisker bar is the result of the analysis of between 10 and 18 neurons; data were pooled from seven independent experiments. Boxes extend from the 25 th to 75 th percentiles of the data, and whiskers extend from the minimum value to the maximum value. For ASEL, a two-way ANOVA revealed no interaction between conditioning time and condition $(F(2,70)=2.346, p=0.1033)$. For ASER, a two-way ANOVA revealed a significant interaction between conditioning time and condition $(F(2,62)=12.94, p<0.0001)$. $\boldsymbol{B}$, Photomicrographs of transgenic animals expressing GFP-tagged CMK-1fused to an exogenous NES (top) or NLS (bottom). The genotypes analyzed were as follows: cmk-1(ok287);cmk-1p::cmk-1(WT)::GFP, cmk-1(ok287);cmk-1p::cmk-1(WT)::NES::GFP, and cmk-1(ok287);cmk-1p::cmk-1(WT)::GFP::NLS. Scale bar, 20 $\mu$ m. C, Proportion of neurons expressing more CMK-1 in the cytoplasm $(C>N)$, the nucleus $(C<N)$, or similar levels in both compartments $(C=N)$. The genotypes analyzed were as follows: cmk-1(ok287);cmk-1p::cmk-1(WT)::GFP, cmk-1(ok287);cmk-1p::cmk-1(WT)::NES::GFP, and cmk-1(ok287);cmk-1p:::cmk-1(WT)::GFP::NLS. Each bar represents between 14 and 37 neurons. D, Behavior of cmk-1(ok287) mutants expressing (MK-1 fused to an NES or an NLS. The genotypes analyzed were as follows: cmk-1(ok287), cmk-1(ok287);cmk-1p:::cmk-1(WT)::NES::GFP, (Figure legend continues.) 
CaM kinases are known to phosphorylate transcription factors such as CREB and HSF-1, thereby regulating activity-dependent changes in gene expression (Sheng et al., 1991; Eto et al., 1999; Holmberg et al., 2001; Wayman et al., 2008). However, loss-offunction mutants in CREB, $c r h-1$, or HSF-1, $h s f-1$, responded to salt conditioning like wild-type animals (Fig. 2D). Thus, saltaversive learning is independent of CREB and HSF-1. Similar results were observed in some (Satterlee et al., 2004; Yu et al., 2014; Kobayashi et al., 2016), but not all (Moss et al., 2016), forms of $c m k$-1-dependent plasticity studied to date. Collectively, these data implicate a pathway whereby CMK-1 is activated via phosphorylation by CKK-1 and subsequently phosphorylates unknown substrates responsible for salt-aversive learning (Fig. 2E).

\section{CMK-1 acts in ASE neurons to regulate salt learning}

Because CMK-1 is expressed broadly throughout the worm nervous system (Kimura et al., 2002; Satterlee et al., 2004) and proper chemotaxis behavior depends not only on salt-sensing, but also on neural circuits linking sensory events to behavior, we sought to determine the neuronal locus of $c m k-1$ action vis-à-vis saltaversive learning. Given that prior work has shown that CMK-1 acts in primary sensory neurons to regulate behavioral (Schild et al., 2014; Yu et al., 2014) and developmental (Neal et al., 2015) plasticity, we focused on the primary salt-sensing neurons, ASER and ASEL. First, we verified that CMK-1 is expressed in the ASE neurons by coexpressing CMK-1::GFP with a known ASE marker (Fig. 3A). Next, we generated and tested transgenic lines that could restore wild-type CMK-1 protein to selected neurons in a cmk-1-null mutant background. We found that expressing wildtype CMK-1 protein under the control of the ASE-specific $f l p-6$ promoter was sufficient to restore salt-aversive learning to $\mathrm{cmk}-1$ (ok287) mutants. The ability to rescue salt-aversive learning appears to be specific to $f l p-6 p$, because expressing CMK-1 under the control of the mechanosensory neuron-specific mec-3p or the AWC chemosensory-neuron specific ceh-36Dp promoters failed to rescue wild-type behavior (Fig. 3B). These findings indicate that not only is CMK-1 expressed in the ASE neurons, but also that CMK-1 functions in a cell-autonomous manner in these sensory neurons to control salt-aversive learning behavior.

Having determined that CMK-1 acts in the two ASE neurons, we next tested whether or not expressing CMK-1 in only the ASEL or ASER neuron might be sufficient to restore salt-avoidance behavior to $c m k$-1 (ok287)-null mutants. To achieve this goal, we used the ASEL-specific $g c y-6$ promoter and the ASER-specific $g c y$-5 promoter (Yu et al., 1997) to drive expression of wild-type CMK-1. In two independent transgenic lines, we found that expressing wild-type CMK-1 in either the ASEL or the ASER neuron was sufficient to

\footnotetext{
(Figure legend continued.) and cmk-1(ok287);cmk-1p:::cmk-1(WT)::GFP::NLS. Each circle is the result of a single assay and bars indicate mean $\pm S D$. Data were pooled from at least two independent experiments, where each experiment consisted of three to six plates per condition. A two-way ANOVA across all genotypes and conditions revealed a significant interaction between genotype and condition $(F(3,124)=29.21, p<0.0001)$. $\boldsymbol{E}$, A gain-of-function allele of cmk-1 behaves like wild-type. Each circle represents the results of a single assay and bars indicate mean \pm SD. Data were pooled from least two independent experiments, where each experiment consisted of three to six plates per condition and was blinded to genotype. A twoway ANOVA revealed a nonsignificant interaction between genotype and condition $(F(1,20)=0.6954, p=0.4142)$. The schematic (bottom) shows the protein predicted to be encoded by $\mathrm{cmk}-1(\mathrm{pg} 58)$ and is adapted from (Schild et al., 2014). Sidak's posthoc tests were used to assess the effect of conditioning and to compare genotypes in panels $\boldsymbol{A}, \boldsymbol{D}$ and $\boldsymbol{E}$. The results are indicated by asterisks: ${ }^{* * *}$ and ${ }^{* * *}$ denotes $p<0.001$ and $p<0.0001$ and ns denotes not significant.
}

partially restore salt-avoidance behavior (Fig. 3C and Fig. 3-1, available at https://doi.org/10.1523/JNEUROSCI.1611-17.2018. f3-1). Expressing CMK-1 from both the $g c y-6$ and $g c y-5$ promoters had a similar effect. These findings reinforce the idea that CMK-1 functions in the ASE neurons to enable salt-aversive learning, but do not implicate either of the two ASE neurons as a dominant factor in this process.

We then examined how the CMK-1 protein responded to salt stimulation in these neurons. Previous studies reported nuclearcytoplasmic shuttling of CMK-1 in primary sensory neurons (Schild et al., 2014; Yu et al., 2014; Neal et al., 2015) and in concert with modulation of glutamate receptor expression (Moss et al., 2016). We sought to observe the subcellular localization of CMK-1 in the cell bodies of the two ASE neurons by imaging a fusion between CMK-1 and GFP. The fusion protein is sufficient to restore wild-type salt-aversive learning to $c m k-1$ (ok287) mutants (Fig. 1D), indicating the fusion protein functions like the wildtype protein under these conditions. Before conditioning, the CMK-1 protein localizes primarily to the cytoplasm of both ASE neurons (Fig. 4A). We did not detect any changes in the nuclearcytoplasmic ratio in the ASEL or ASER cell body during conditioning for between 10 and $180 \mathrm{~min}$ in $20 \mathrm{~mm} \mathrm{NaCl}$ (Fig. 4A). Although conditioning in salt-free buffer transiently increased the localization of CMK-1 throughout cell body in ASER neurons, the significance of this finding for salt-aversive learning is unclear because learning does not occur under these conditions. We next expressed forms of CMK-1 targeted to the nucleus or the cytoplasm, thanks to the adjunction of ectopic nuclear localization signal (NLS) or nuclear export signal (NES), respectively (Fig. 4B,C). In both cases, the learning defect of $c m k-1$ ( ok287) mutants was rescued (Fig. 4D). Moreover, a mutant that encodes a form of CMK-1 with a deleted predicted NES (and a portion of the autoinhibitory domain), cmk-1 (pg58), exhibited normal salt learning (Fig. 4E). Collectively, these findings suggest that neither the precise localization of CMK-1 nor nuclear-cytoplasmic shuttling in the ASE cell bodies are essential for salt-aversive learning.

\section{Calcium signals in sensory dendrites, cell bodies, and axons of ASE neurons}

The two ASE neurons are bilaterally-symmetric, bipolar chemosensory neurons whose cell bodies and dendrites lie on the left and right sides of the animal and whose axons overlap in the nerve ring (Fig. 5A). Despite their similar appearance, the two ASE neurons are functionally distinct: ASEL detects salt increases and ASER is activated by salt decreases (Hobert, 2014). Ample evidence supports this general description of chemosensory responses in the ASE cell bodies (Tomioka et al., 2006; Suzuki et al., 2008; Ortiz et al., 2009; Thiele et al., 2009; Oda et al., 2011; Kunitomo et al., 2013; Luo et al., 2014; Rabinowitch et al., 2014), but less is known about how the calcium signals generated in ASE dendrites, cell bodies, and axons compared to one another. We sought to fill this gap in knowledge by recording salt-evoked calcium signals in the sensory endings (dendrites), cell bodies, and axons of the wild-type ASE neurons. To reach this goal, we took advantage of mosaic expression of the $y x E x 738$ [flp-6p:: GCaMP3; ttx-1p::GFP; unc-122p::GFP] transgene and recorded responses to pulses of sodium chloride salt in the ASEL dendrites, cell bodies, and axons independently of the ASER neuron and vice versa. Figure $5 B$ shows raster plots of salt-evoked calcium signals measured in all three compartments (sensory dendrite, cell body, axon) of the ASEL and ASER neurons. Each line in the raster plot represents calcium signals as a function of time in the indicated compartment. 
A

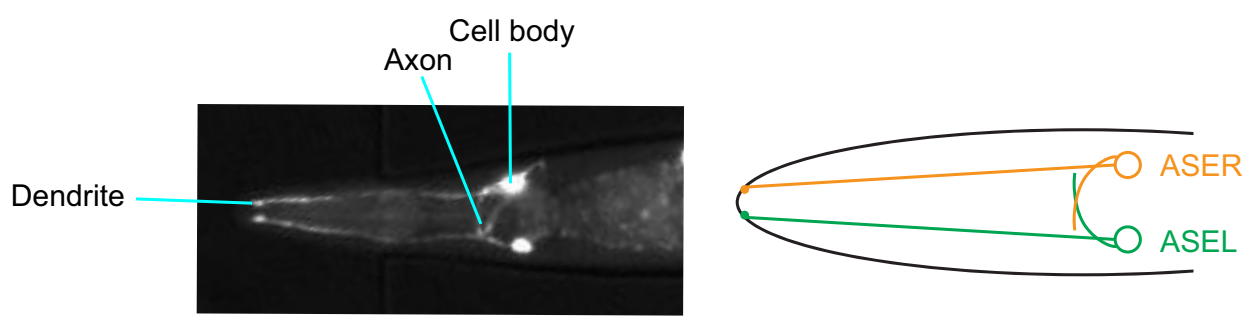

B
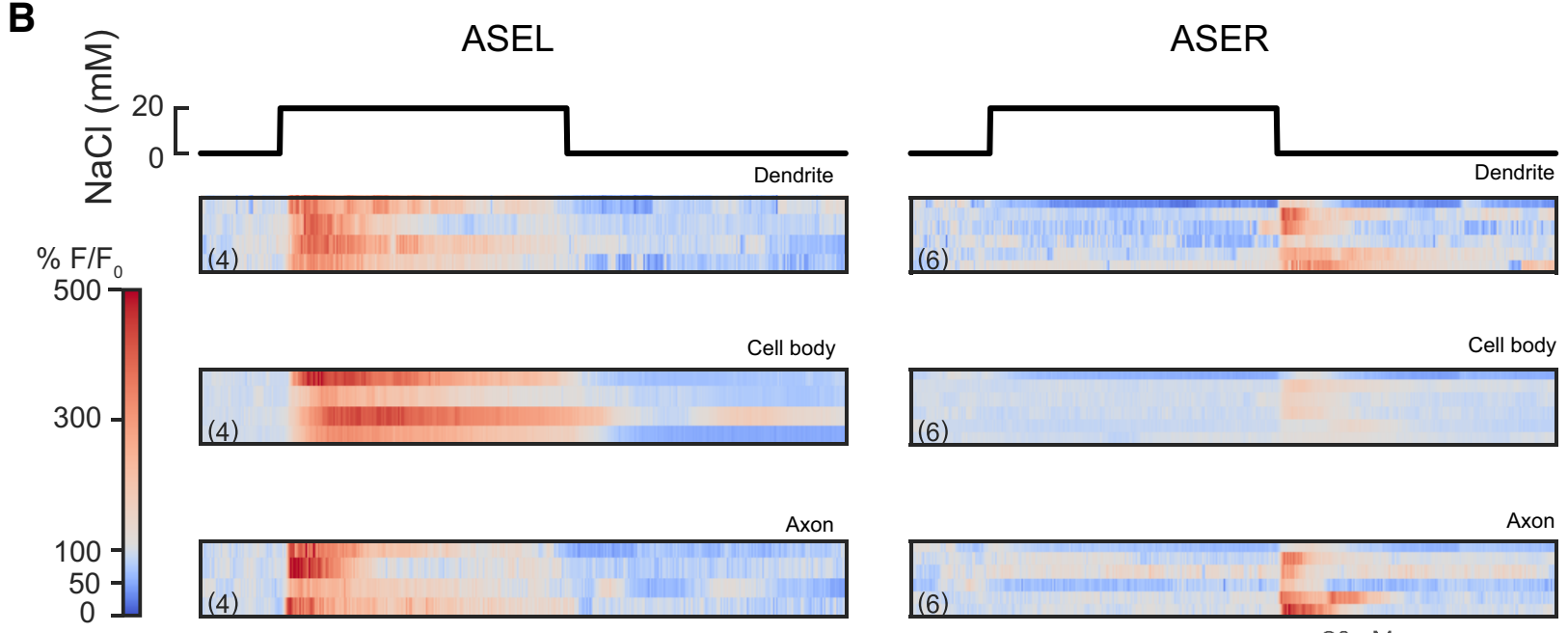

$(4)$

C
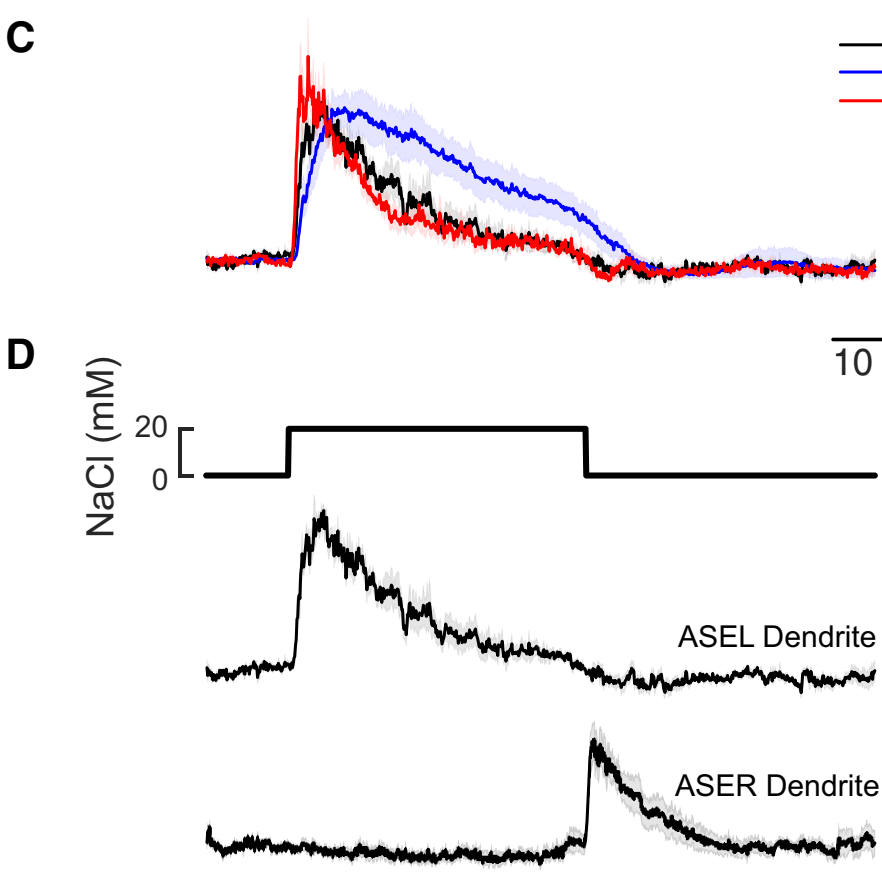

$\int_{\Delta \mathrm{s}}^{100 \%}$

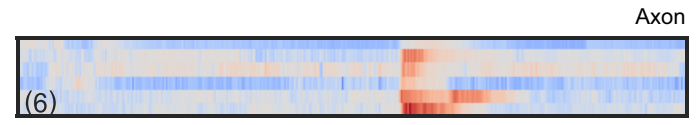

$20 \mathrm{mM}$

Cell body

(6)

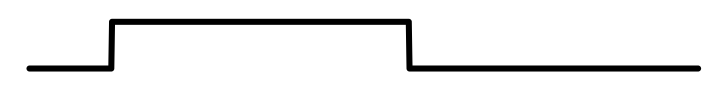

Figure 5. Calcium signals in wild-type ASEL and ASER dendrites, cell bodies, and axons. A, Image and schematic of ASE neurons. Left, Wild-type worms expressing yxEx738 [flp-6p::GCaMP3 + unc-122p::GFP]. The dendrite tips, cell bodies, and axons are easily visualized, allowing for calcium imaging of different regions of these cells. Right, Schematic of ASE neurons. Neurite tips and cell bodies of ASEL and ASER are readily distinguishable from each other, but axons overlap in the part of the neurons that synapse onto the nerve ring of the worm. $\boldsymbol{B}$, Raster plots of GCaMP3 fluorescence as a function of time in the dendrite, cell body, and axon of the ASEL (left) and ASER (right) neurons of wild-type worms conditioned in $0 \mathrm{~mm} \mathrm{NaCl}$ and no food and exposed to a $40 \mathrm{~s}$ pulse of $20 \mathrm{~mm}$ $\mathrm{NaCl}$. Color indicates fluorescence relative to prestimulus control values, $\Delta F / F_{0}$ : white indicates values similar to $F_{0}$, red and blue indicate increases and decreases, respectively. All recordings were obtained in animals expressing yxEx738 in ASEL or ASER, but not in both neurons and the number of recordings is indicated in parentheses. $C$, Average calcium signals in the dendrite, cell body, and axon of the ASEL (left) and ASER (right) neurons of wild-type worms derived from the data shown in $\boldsymbol{B}$, normalized to prestimulus values. Lines indicate mean of corresponding traces and light shading indicates \pm SEM. Inset, The average ( \pm SEM) responses recorded in ASER and normalized to values recorded before the down-step. $D$, Normalized fluorescence of calcium traces in dendrites of the ASE neurons of wild-type worms conditioned in $0 \mathrm{~mm} \mathrm{NaCl}$ and no food and exposed to up-step ( $0-20 \mathrm{~mm}$ ) and down-step ( $20-0 \mathrm{~mm})$ of NaCl (left; same data as in C). Average of calcium traces in dendrites of ASEL and ASER (right). Lines in normalized fluorescence plots indicate mean of corresponding traces and light shading indicates \pm SEM. 
A
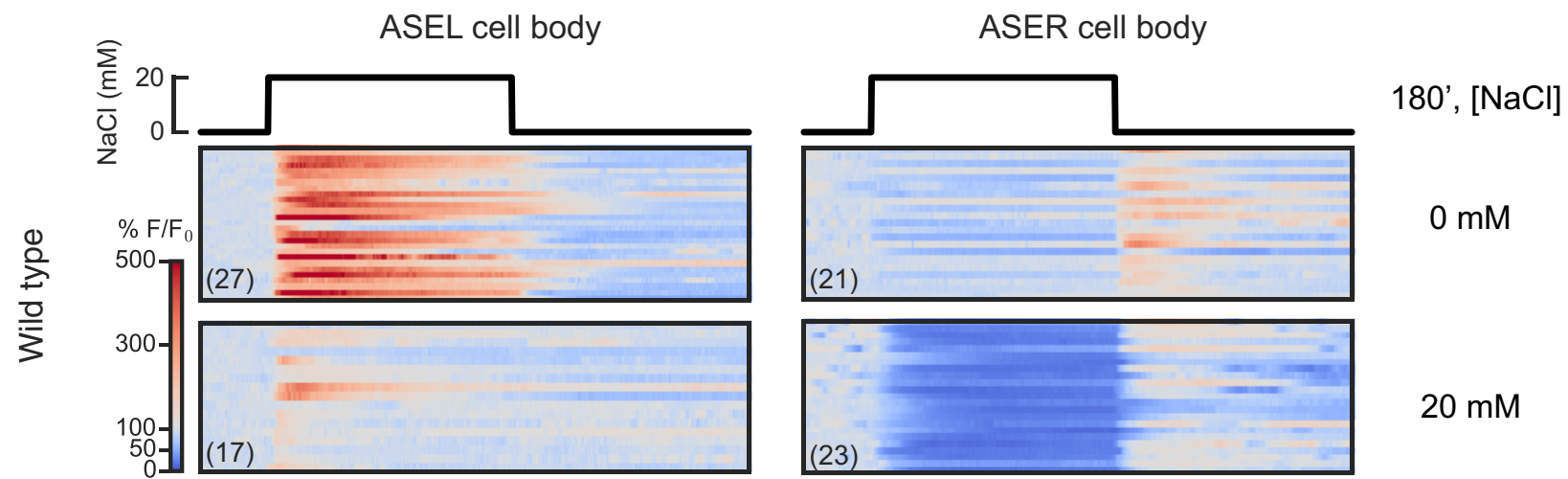

B

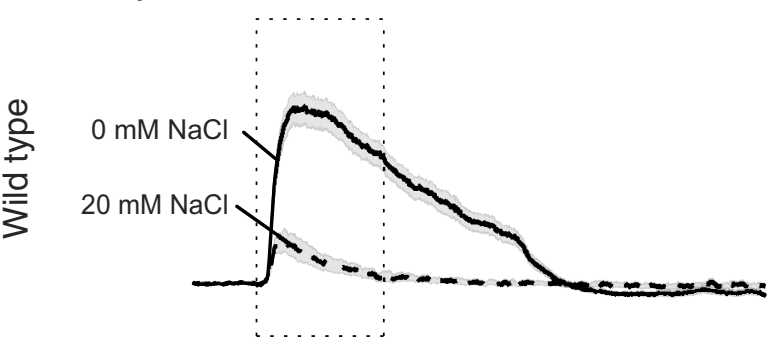

(21)

C
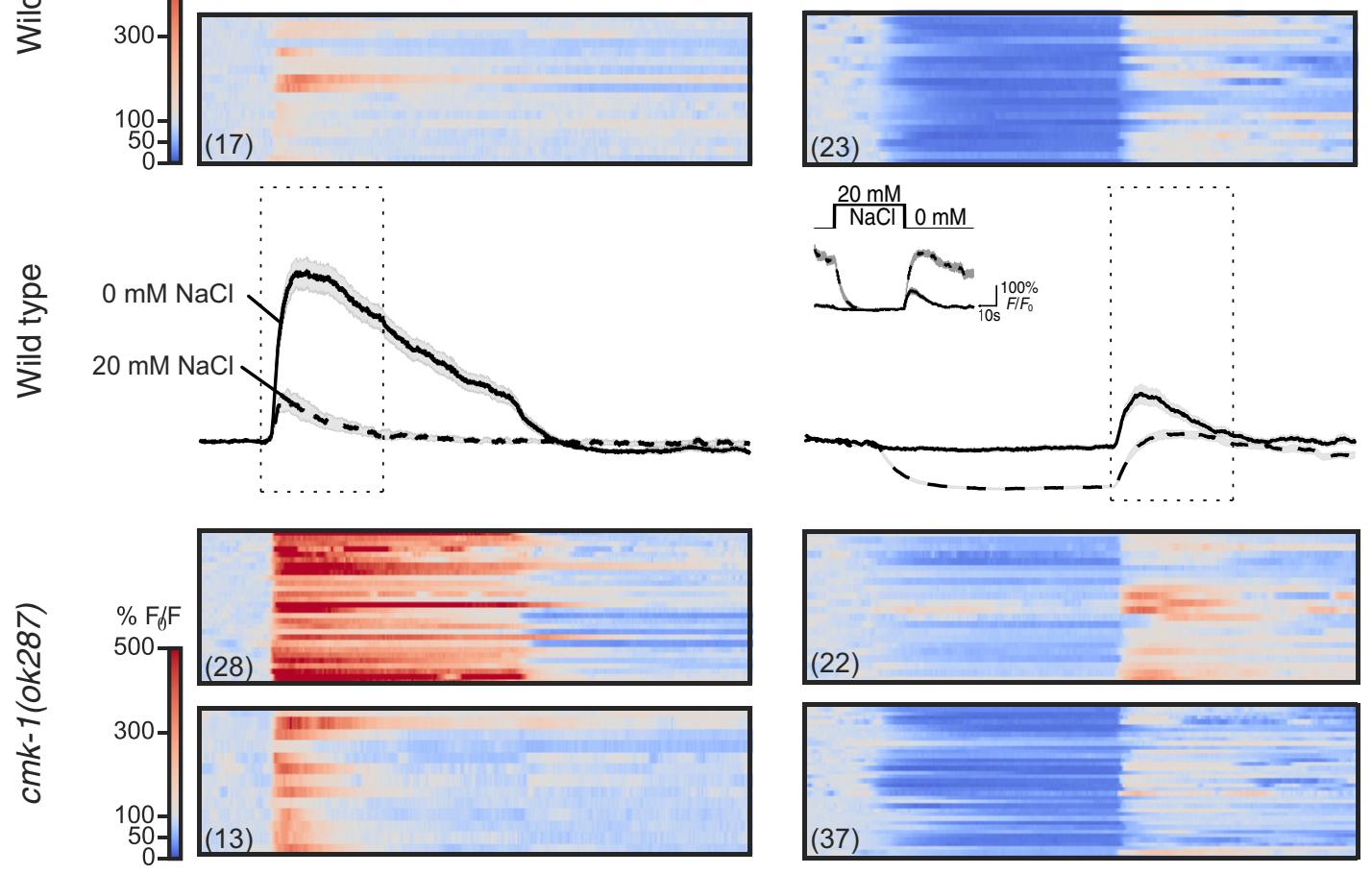

$20 \mathrm{mM}$

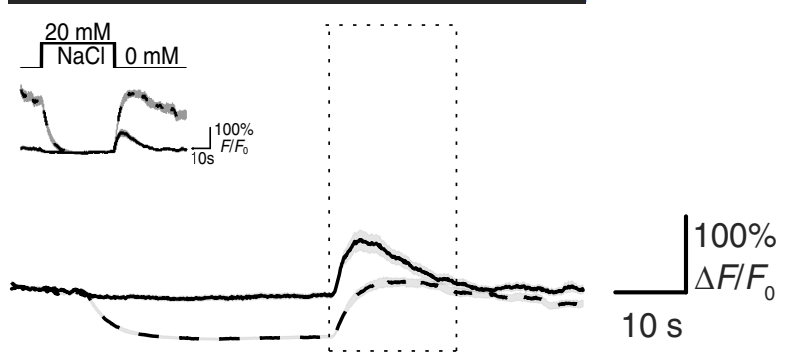

D
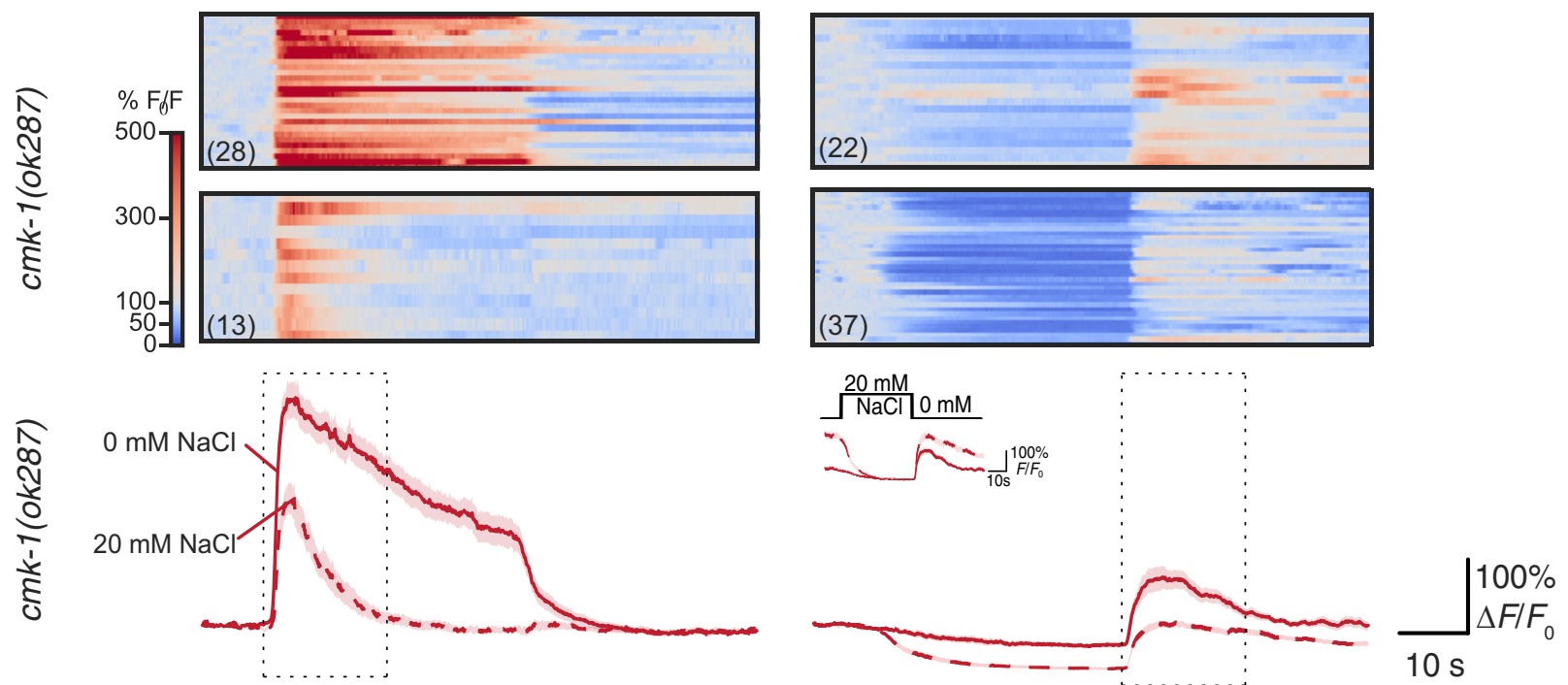

E
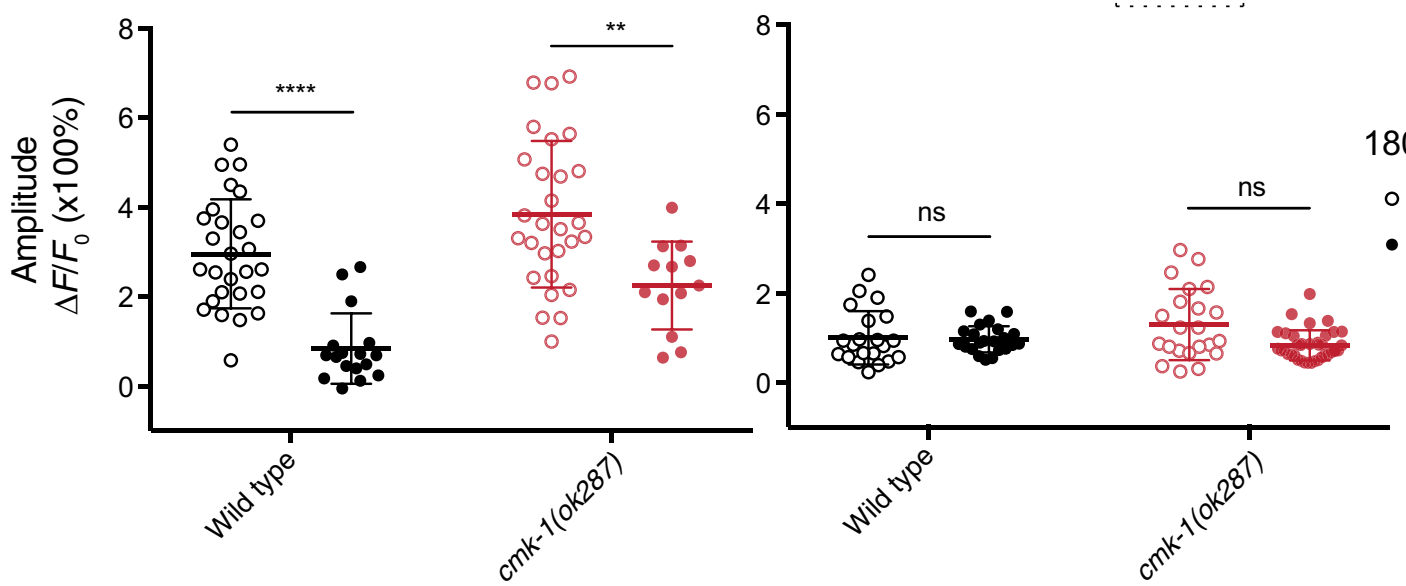

Figure 6. Salt conditioning modifies salt-evoked calcium signals in wild-type and cmk-1 mutant ASER and ASEL cell bodies. A, Raster plots of GCaMP3 fluorescence as a function of time in the cell bodies of the ASEL (left) and ASER (right) neurons of wild-type worms conditioned in 0 or $20 \mathrm{~mm} \mathrm{NaCl}$ and no food. Animals were exposed to $0 \mathrm{~mm} \mathrm{NaCl} \mathrm{saline} \mathrm{and} \mathrm{stimulated} \mathrm{by} \mathrm{a} 40 \mathrm{~s} \mathrm{pulse} \mathrm{of} 20 \mathrm{~mm}$ $\mathrm{NaCl}$. Each horizontal line corresponds to a recording from one cell body; the number of recordings is indicated in parentheses. Color indicates fluorescence relative to prestimulus control values, $\Delta F / F_{0}$ : white indicates control values, red and blue indicate increases and decreases, respectively. $\boldsymbol{B}$, Average calcium signals in the cell bodies of the ASEL (left) and ASER (right) neurons of wild-type worms. The traces are derived from the data shown in $\boldsymbol{A}$. Inset, The waveform generated when signals generated by ASER cell body are normalized to values measured (Figure legend continues.) 
Salt pulses produced calcium transients synchronized to the up-step in ASEL and the down-step in ASER in all three subcellular domains (Fig. 5C). The inset shows ASER calcium transients normalized to the fluorescence recorded just before the downstep. Although dendritic endings and axons lie on opposite sides of the cell body and are at least $100 \mu \mathrm{m}$ apart from one another, the time course of their calcium signals were indistinguishable from one another. Responses developed more slowly and lasted longer in cell bodies than in dendrites or axons, a result that could be explained by the cell body's larger volume acting as a low-pass filter for calcium signals. These observations are compatible with the electrically compact nature of the ASE neurons (Goodman et al., 1998) and a lack of distinct, subcellular calcium domains in these cells (Kuramochi and Doi, 2017).

We averaged the mean responses of ASEL and ASER axons to generate a prediction for the waveform expected in animals expressing a calcium indicator in both axons (Fig. 5D). These reconstructed traces recapitulate the phasic responses we detected in animals expressing in both ASE axons (see Fig. 7D). With this analysis, we confirm that ASEL functions as an ON cell that is sensitive primarily to salt up-steps whereas ASER functions as an OFF cell that responds primarily to salt down-steps. We establish that signals recorded in sensory dendrites, but not cell bodies, mirror the stimulus-induced calcium waveforms present in axons. We further propose that signals recorded from the nerve ring of animals expressing calcium indicator in both neurons can be interpreted as the average of the axonal signals generated independently by ASEL and ASER.

\section{Salt conditioning alters salt-evoked calcium signals in wild-type and $c m k-1$ mutant ASEL and ASER cell bodies}

Calcium signals recorded in the cell bodies of specific neurons are a common proxy for neuronal activity and have been used to understand how acclimation affects sensory signaling in several C. elegans behaviors (Clark et al., 2006; Biron et al., 2008; Yu et al., 2014). Following this approach, we analyzed chemosensory re-

\section{$\leftarrow$}

(Figure legend continued.) before the down-step. Lines indicate the mean and light shading indicates \pm SEM; solid and dashed lines indicate data collected from animals conditioned in 0 and $20 \mathrm{~mm} \mathrm{NaCl}$, respectively. Figure 6-1 (available at https://doi.org/10.1523/JNEUROSCl. 1611-17.2018.f6-1) shows the response of ASEL and ASER cell bodies to transient decreases in external salt (from 20 to $0 \mathrm{~mm}$ ) recorded from animals conditioned in 0 and $20 \mathrm{~mm} \mathrm{NaCl}$. C, Raster plots of GCaMP3 fluorescence as a function of time in the cell bodies of the ASEL (left) and ASER (right) of $\mathrm{cmk}-1$ (ok287) worms conditioned in 0 or $20 \mathrm{~mm} \mathrm{NaCl}$ and no food and exposed to a $40 \mathrm{~s}$ pulse of $20 \mathrm{~mm} \mathrm{NaCl}$. Each horizontal line corresponds to a recording of a single cell body; the number of recordings is indicated in parentheses. Color indicates fluorescence relative to prestimulus control values, $\Delta F / F_{0}$ : white indicates control values, red and blue indicate increases and decreases, respectively. $\boldsymbol{D}$, Average fluorescence of calcium traces in cell bodies of the ASEL (left) and ASER (right) neurons of $\mathrm{cmk}$-1 (ok287) worms conditioned in 0 or $20 \mathrm{~mm} \mathrm{NaCl}$ and no food and exposed to a 40 s pulse of $20 \mathrm{~mm} \mathrm{NaCl}$. The traces are derived from the data shown in C. Lines indicate the mean and light shading indicates \pm SEM. $E$, Maximum amplitude of the change in calcium signals recorded in cell bodies of the ASEL (left) and ASER (right) neurons of wild-type and $\mathrm{cmk}$-1 ( 0 k287) worms conditioned in 0 or $20 \mathrm{~mm} \mathrm{NaCl}$ and no food. The amplitude was extracted from calcium traces by subtracting the average signal of $10 \mathrm{~s}$ before the outlined region from the maximum signal in the region outlined by dotted line in the normalized fluorescence plots in $\boldsymbol{B}$ and $\boldsymbol{D}$. Data were analyzed using the Wilcoxon rank sum test for nonparametric data, where $p$ values were adjusted for multiple hypothesis testing using the BenjaminiHochberg method. There was a significant effect of salt conditioning upon $0 \mathrm{~N}$ responses in the ASEL body in both wild-type and cmk-1 (ok287) animals (wild-type $p<0.0001$, cmk-1 (ok287) $p<0.01$ ). A significant effect of salt conditioning upon OFF responses in the ASER cell bodies was detected only in $c m k-1$ (ok287) mutants: wild-type $p=0.5728$, cmk-1(ok287) $p=$ $0.0691)$. ${ }^{* *}$ and ${ }^{* * * *}$ indicate $p<0.01$ and $p<0.0001$, respectively and ns denotes not significant. sponses in ASE cell bodies of wild-type and $c m k-1$ mutants conditioned in the absence or presence of salt (Fig. 6). In wild-type worms, salt conditioning decreased ON responses in ASEL cell bodies (Fig. $6 A, B, E$, left). This effect of salt conditioning on wildtype ASEL chemosensory responses was also evident in recordings of the response to a $40 \mathrm{~s}$ decrease in salt from 20 to $0 \mathrm{~mm}$ (Fig. 6-1 A, available at https://doi.org/10.1523/JNEUROSCI.1611-17. 2018.f6-1). Consistent with its function as an OFF cell, salt application had little effect on calcium levels and down-steps evoked an increase in calcium in ASER cell bodies (Fig. 6A, $B, E$, right and Fig. 6-1 $B$, available at https://doi.org/10.1523/JNEUROSCI. 1611-17.2018.f6-1). This OFF response was also evident when we normalized to fluorescence levels recorded just before the downstep (Fig. 6B, inset). Following salt conditioning, however, calcium was suppressed during salt application and OFF responses were maintained in the ASER cell body (Fig. 6A,B). If we controlled for the salt-induced decrease in calcium signals by normalizing to fluorescence values at the end of the salt pulse, it appears that salt conditioning increases the amplitude of OFF responses (Fig. 6B, inset). These observations agree with prior studies (Oda et al., 2011) and indicate that salt conditioning modulates the chemosensory responses in both ASE cell bodies.

Although $c m k$-1 (ok287) mutants fail to develop salt avoidance (Fig. 1C) and this deficit is rescued by expression of wild-type CMK-1 in the ASE neurons (Fig. $3 B, C$ ), salt-evoked calcium signals in $c m k-1$ (ok287) mutant ASEL and ASER cell bodies were similar to those observed in wild-type (Fig. $6 E$ ). As found in wild-type animals, salt conditioning decreased $\mathrm{ON}$ responses to salt pulses in the ASEL neurons in $c m k-1$ mutants (Fig. $6 C, D$, left) and had only minor effects on OFF responses in the ASER neurons in $c m k-1$ mutants (Fig. $6 C, D$, right). A subtle effect of loss of $c m k-1$ on ASER signals is apparent in our data: salt application decreases ASER GCaMP3 signals relative to prestimulus levels in animals conditioned in the absence and presence of $20 \mathrm{~mm}$ salt (Fig. $6 C, D$, right). The net result of this effect is a smaller effect of salt conditioning on ASER OFF responses and is evident in plots controlling for the salt-induced decrease (Fig. 6D, inset).

Peak ON responses in the ASEL neurons decrease in both wild-type and $c m k-1$ ( ok287) ASEL cell bodies upon conditioning (Fig. 6E, left). As noted above, salt conditioning had minor effects on peak OFF responses in wild-type and $c m k-1$ ( ok287) ASER cell bodies (Fig. 6E, right), unless the stimulus-induced decrease in GCaMP signaling was considered. In the latter situation, salt conditioning increases OFF responses in both wild-type and $c m k-1$ mutant ASER cell bodies and this effect is weaker in $c m k-1 \mathrm{mu}-$ tants. These findings indicate that ASEL and ASER neurons retain their function as ON and OFF cells, respectively, and that the modulation of ASE chemosensory responses following salt conditioning is weakened by loss of $c m k-1$ function.

\section{The effect of salt conditioning on chemosensory calcium signals in ASEL and ASER dendrites and axons is regulated by cmk-1}

Given that the response dynamics in ASE cell bodies are slower than signals observed at the tip of the ASE dendrites and along their axons (Fig. 5), we also determined the effect of salt conditioning and loss of $c m k-1$ function on calcium signals recorded in these subcellular domains. In particular, we reasoned that a closer look at the axons that harbor all of ASE's synaptic connections to the nervous system might provide additional insight into how loss of $c m k-1$ function in ASE neurons contributes to the deficit in salt-aversive learning. Consistent with current and prior findings in the cell bodies, ASEL sensory endings detect up-shifts in salt 

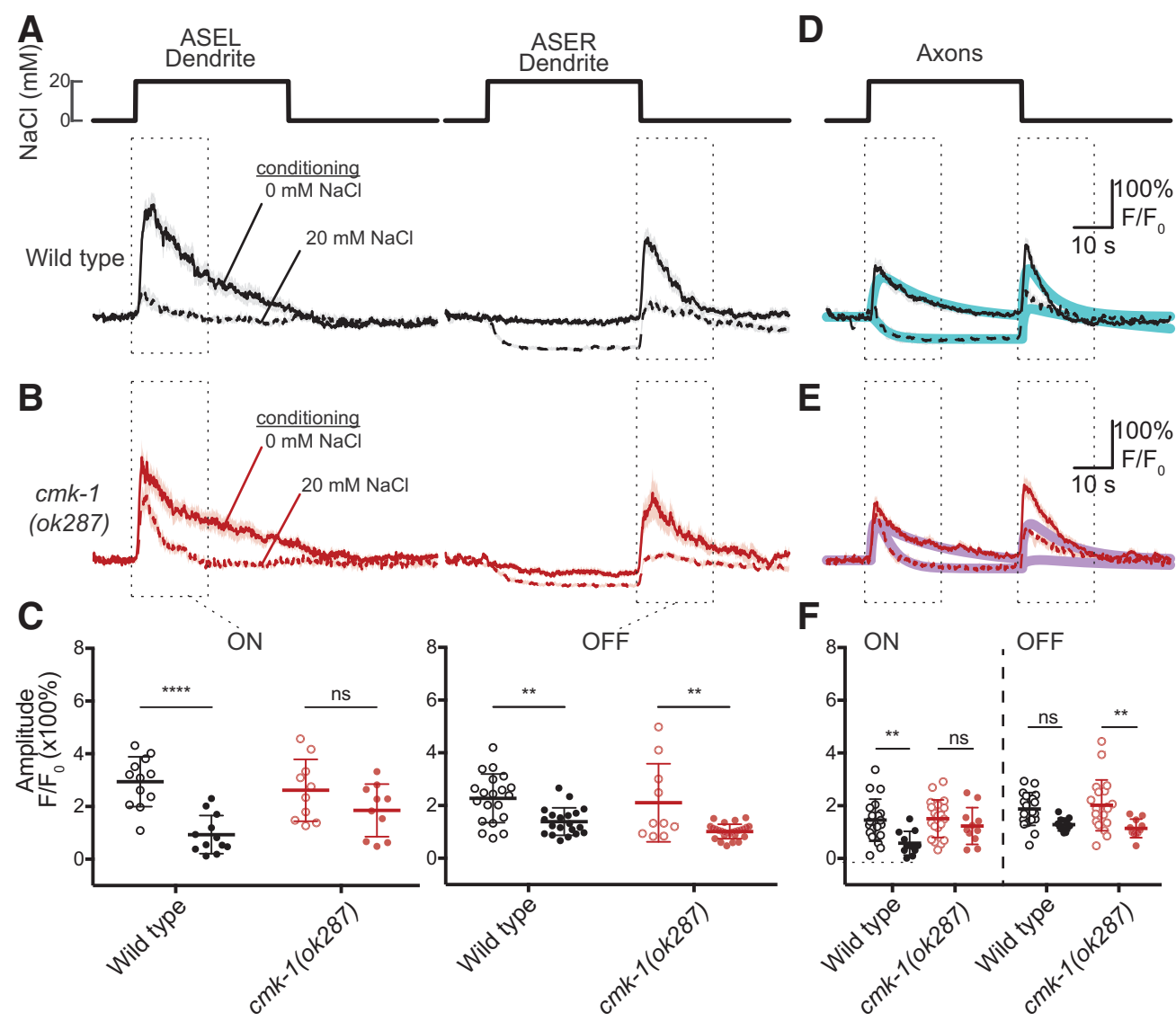

Figure 7. CMK-1 is required for the effect of salt conditioning on chemosensory calcium signals in dendrites and axons of ASE sensory neurons. A, Average chemosensory calcium signals recorded from ASEL (left) and ASER dendrites (right) in wild-type worms conditioned in 0 or $20 \mathrm{~mm} \mathrm{NaCl}$ and no food and exposed to a $40 \mathrm{~s}$ pulse of $20 \mathrm{~mm} \mathrm{NaCl}$. Lines indicate the mean and light shading indicates \pm SEM of at least 12 recordings. Figure 7-1A (available at https://doi.org/10.1523/JNEUROSCI.1611-17.2018.f7-1) shows the individual replicates for ASEL (left) and ASER (right) dendrites. $B$, Average chemosensory calcium signals recorded from ASEL (left) and ASER dendrites (right) in cmk-1 (ok287) worms conditioned in 0 or $20 \mathrm{~mm} \mathrm{NaCl} \mathrm{and} \mathrm{no} \mathrm{food} \mathrm{and} \mathrm{exposed} \mathrm{to} \mathrm{a} 40 \mathrm{~s}$ pulse of $20 \mathrm{~mm} \mathrm{NaCl}$. Lines indicate the mean and light shading indicates \pm SEM of at least 10 recordings. Figure 7-1B (available at https://doi.org/10.1523/JNEUROSCl.1611-17.2018.f7-1) shows the individual replicates for ASEL (left) and ASER (right) dendrites. C, Maximum amplitude of ON (left) and OFF (right) responses in dendrites of ASE neurons of wild-type and cmk-1(ok287) worms conditioned in $0 \mathrm{~mm} \mathrm{NaCl}$ (open circles) or $20 \mathrm{~mm} \mathrm{NaCl}$ (closed circles). Maximum amplitude was extracted from calcium traces by subtracting the average signal measured $10 \mathrm{~s}$ before the outlined region from the maximum signal in the region outlined by dotted line in the normalized fluorescence plots in $\boldsymbol{A}$ and $\boldsymbol{B}$. For the ASEL dendrite, a two-way ANOVA revealed a significant interaction between genotype and condition $(F(1,40)=4.546, p=0.0392)$. For the ASER dendrite, a two-way ANOVA failed to detect an interaction between genotype and condition $(F(1,73)=0.303, p=$ $0.5837)$. $\boldsymbol{D}$, Average chemosensory calcium signals recorded from ASE axons in wild-type worms conditioned in 0 or $20 \mathrm{~mm} \mathrm{NaCl}$ and no food and exposed to a $40 \mathrm{~s} \mathrm{pulse} \mathrm{of} 20 \mathrm{~mm} N a C l$. Lines indicate the mean and light shading indicates \pm SEM of at least 11 recordings. Figure 7-1A (right; available at https://doi.org/10.1523/JNEUROSCl.1611-17.2018.f7-1) shows the individual replicates of wild-type axon recordings. The solid cyan lines are a weighted average of the mean traces of the ASEL and ASER dendrites, which were fitted individually $\left[d_{A S E L}(t), d_{A S E R}(t)\right.$, as described in Materials and Methods]. The weighting factor, $d_{\text {Axon }}(t)=A^{*} d_{\text {ASEL }}(t)+(1-A)^{*} d_{A S E R}(t)$, was estimated by minimizing the $r^{2}$ of the difference between $d_{\text {Axon }}(t)$ and the mean fluorescence signal measured from the axon (black line). The weighting factors were 0.4 and 0.29 for 0 and $20 \mathrm{~mm} \mathrm{NaCl}$ conditioned animals. $E$, Average chemosensory calcium signals in the axons of ASE neurons in $\mathrm{cmk}-1$ ( $0 \mathrm{k} 287$ ) worms conditioned in 0 or $20 \mathrm{~mm} \mathrm{NaCl}$ and no food and exposed to a 40 s pulse of $20 \mathrm{~mm} \mathrm{NaCl}$. Lines indicate the mean and light shading indicates \pm SEM of at least 11 recordings. Figure $7-1 B$ (right; available at https://doi.org/10.1523/JNEUROSCI.1611-17.2018.f7-1) shows the individual replicates for $\mathrm{cmk}$ - 1 mutant axon recordings. The solid magenta lines are a weighted average of fits to the mean traces of the ASEL and ASER dendrites $\left[d_{A S E L}(t), d_{A S E R}(t)\right.$; as described in Materials and Methods]. The weighting factor, $d_{A x o n}(t)=A^{*} d_{A S E L}(t)+(1-A) * d_{A S E R}(t)$, was estimated by minimizing the $r^{2}$ of the difference between $d_{A x o n}(t)$ and the mean fluorescence signal measured from the axon. The weighting factors were 0.47 and 0.67 for 0 and $20 \mathrm{~mm} \mathrm{NaCl}$ conditioned animals, respectively. $\boldsymbol{F}$, Maximum amplitude of $\mathrm{ON}$ (left) and OFF (right) responses in the ASE axons recorded from wild-type and cmk-1(ok287) worms conditioned in $0 \mathrm{~mm} \mathrm{NaCl}$ (open circles) or $20 \mathrm{~mm} \mathrm{NaCl}$ (closed circles). Maximum amplitude was extracted from calcium traces by subtracting the average signal of 10 s before the outlined region from the maximum signal in the region outlined by dotted line in the normalized fluorescence plots in $\boldsymbol{D}$ and $\boldsymbol{E}$. A two-way ANOVA failed to reveal an interaction between genotype and condition $(F(3,116)=1.203, p=0.3119)$. Sidak's posthoc tests were used to assess the effect of conditioning and to compare genotypes in panels $C$ and $\boldsymbol{F}$. The results are indicated by asterisks: ${ }^{* *}$ and ${ }^{* * *}$ denote $p<0.01$ and $p<0.0001$, respectively and ns denotes not significant.

and ASER endings detect downshifts. In wild-type worms, the peak amplitude of the ON response in ASEL dendrites and the OFF response in ASER dendrites decreased after salt conditioning (Fig. $7 A$ and Fig. $7-1 B$, left and center, available at https:// doi.org/10.1523/JNEUROSCI.1611-17.2018.f7-1). This effect of conditioning was diminished in $c m k-1$ mutant ASEL dendrites (Fig. $7 B$ and Fig. 7-1 B, left and center, available at https://doi.org/ 10.1523/JNEUROSCI.1611-17.2018.f7-1). We observed a qualitatively similar, but less dramatic effect upon salt conditioning in cmk-1 mutant ASER dendrites. We summarized these results by comparing peak calcium values observed at the onset (ASEL) and offset (ASER) of the salt pulse (Fig. 7C). A two-way ANOVA revealed a significant effect of conditioning on the amplitude of ON responses in ASEL $(F(1,40)=22.67, p<0.0001)$ and an interaction between genotype (wild-type vs $c m k-1$ ) and conditioning in $\operatorname{ASEL}(F(1,40)=4.546, p=0.0392)$. Although there was also a significant effect of conditioning on the amplitude of OFF responses in $\operatorname{ASER}(F(1,73)=27.72, p<0.0001)$, there was no detectable interaction between genotype (wild-type vs $c m k-1$ ) and conditioning. In summary, loss of $c m k-1$ function blunts the 
ability of salt conditioning to enhance ON responses in ASEL dendrites but has little or no effect on OFF responses in ASER dendrites. The subcellular locus of the defect in signaling remains ambiguous, however, because the ASE neurons are isopotential (Goodman et al., 1998) and appear to lack calcium subdomains (Kuramochi and Doi, 2017).

Finally, we analyzed how conditioning in $20 \mathrm{~mm} \mathrm{NaCl}$ and loss of $c m k-1$ function affected responses in the overlapping ASE axons (Fig. 7D-F), which are responsible for transmitting changes in sensory neuron activity to postsynaptic neurons. Conditioning wild-type animals in $20 \mathrm{~mm} \mathrm{NaCl}$ had two main effects on axonal calcium signals in ASE neurons. First, responses to salt up-steps are transformed from a transient increase in axonal calcium to a sustained decrease in calcium (Fig. $7 D$ and Fig. 7-1 A, right, available at https://doi.org/10.1523/JNEUROSCI.1611-17.2018.f7-1). Second, OFF responses to salt down-steps decreased slightly (Fig. $7 D$ and Fig. 7-1A, right, available at https://doi.org/10.1523/ JNEUROSCI.1611-17.2018.f7-1). Interestingly, in cmk-1 mutant axons, conditioning in $20 \mathrm{~mm} \mathrm{NaCl}$ failed to generate this sustained decrease in calcium in response to salt up-steps (Fig. $7 E$ and Fig. 7-1 B, right, available at https://doi.org/10.1523/JNEUROSCI. 1611-17.2018.f7-1). In addition, $c m k-1$ mutant ASE axons failed to generate wild-type-like conditioned responses to salt downsteps (Fig. 7 E, F and Fig. 7-1 B, right, available at https://doi.org/ 10.1523/JNEUROSCI.1611-17.2018.f7-1). Thus, loss of $\mathrm{cmk-1}$ does not abolish salt-evoked calcium responses in ASE axons upon salt conditioning, but alters the nature of the signal delivered to postsynaptic neurons.

We considered these observations regarding the influence regarding $c m k-1$ on axonal signals in the context of our earlier findings that: (1) chemosensory signals in overlapping axons are consistent with a summation of the signals generated by the ASEL and ASER axons (Fig. 5D) and (2) the chemosensory waveforms recorded in single ASE dendrites are virtually indistinguishable from those recorded in axons (Fig. 5 C). Assuming these relationships hold for $c m k-1$ mutant ASE neurons, we fit chemosensory waveforms measured in wild-type and $c m k-1$ ASER and ASEL dendrites (see Materials and Methods), computed a weighted sum of these waveforms and compared the resulting waveforms to the measured axonal signals. As shown in Figure $7 D$ (cyan curves), this simple strategy was sufficient to recapitulate the effects of conditioning by $20 \mathrm{~mm} \mathrm{NaCl}$ on chemosensory signaling in wild-type animals, reaffirming that conditioning depresses calcium levels during salt pulses. This approach could reproduce the blunted responses to salt up-steps in $c m k-1$ mutants (though it was less successful at reproducing the responses to salt downsteps; Fig. 7E, purple curves).

The fact that $c m k-1$ mutants perform chemotaxis like wildtype animals when conditioned with $0 \mathrm{~mm} \mathrm{NaCl}$ (Fig. 1C) is compatible with our finding that $c m k-1$ (ok287) mutant ASE neurons retain stimulus-evoked calcium signals under these conditions (Figs. 6, 7). The response of $c m k-1$ (ok287) mutant ASE neurons diverged from wild-type upon conditioning in $20 \mathrm{~mm}$ salt, however, and this effect was more pronounced in chemosensory responses recorded in dendrites and axons. The effects on ASE cell bodies were less pronounced, consistent with our inference that chemosensory signals in the ASE cell bodies are filtered compared with these detected in dendrites and axons. Collectively, our data show that although $c m k-1$ loss does not completely abolish the modulation of chemosensory responses by salt conditioning, but does blunt the effect of salt conditioning, especially in the ASEL dendrites and axons.

\section{Discussion}

CMK-1, the C. elegans ortholog of mammalian CaMKI/IV, enables behavioral plasticity related to thermal acclimation and developmental plasticity governed by chemosensation (Satterlee et al., 2004; Schild et al., 2014; Yu et al., 2014; Neal et al., 2015). Here, we demonstrate that CMK-1 is also critical for salt-aversive learning, a process that allows animals to balance the need for salt with the potentially damaging effects of this essential inorganic nutrient. We show that salt-aversive learning depends on CMK-1 expression in the ASE chemosensory neurons, uses an upstream activation pathway involving CKK-1, and requires the kinase activity of CMK-1.

In agreement with a number of prior studies (Suzuki et al., 2008; Ortiz et al., 2009; Thiele et al., 2009; Oda et al., 2011; Rabinowitch et al., 2014), we find that ASEL functions as an ON cell and ASER functions as an OFF cell and that salt conditioning appears to blunt salt-evoked signals. We analyzed stimulusevoked calcium signals in the dendrites, cell bodies, and axons of both ASER and ASEL neurons, extending general knowledge of chemosensory signaling by these neurons. We found that signals in dendrites and axons were indistinguishable from one another and that somatic signals resembled a low-pass filtered version of these signals. These findings are consistent with isopotential electrical signaling by ASE (Goodman et al., 1998) and have implications for relying on somatic calcium signals as a proxy for global activity in the C. elegans nervous system.

Salt-evoked calcium signals in $c m k-1$ mutant ASE dendrites and axons are significantly less sensitive to salt conditioning than they are in their wild-type counterparts, although $c m k-1$ mutant ASE neurons continue to generate both ON and OFF chemosensory responses. Additional work will be needed to distinguish between the effects of $c m k$-1-dependent pathway on chemosensory transduction in dendrites or on presynaptic signaling in axons. This ambiguity follows from prior work demonstrating that the ASE neurons are isopotential (Goodman et al., 1998) and the finding that signaling dynamics are similar in wild-type dendrites and axons. Building on this finding, we hypothesized that signal dynamics in the overlapping axons could be predicted from a weighted sum of the signals in ASEL and ASER dendrites. This simple model recapitulated the principal features of the phasic responses to salt pulses we observed in wild-type ASE axons, but was not adequate to capture the effect of salt conditioning in cmk-1 mutant axons (Fig. 7E). This mismatch favors a model in which CMK-1 affects the sensitivity of transmitter release by ASE axons to salt conditioning, but does not exclude influences on chemosensory transduction or other pathways.

CMK-1 is expressed globally in the C. elegans nervous system (Kimura et al., 2002; Satterlee et al., 2004), but has been shown to act locally in sensory neurons to support plasticity (Schild et al., 2014; Yu et al., 2014; Neal et al., 2015). Its kinase activity is required for plasticity, but CREB, an established CaM kinase substrate, is not essential for the forms of behavioral and developmental plasticity analyzed thus far. At present, we cannot exclude the possibility that CREB acts redundantly with other CMK-1 substrates, including Raf/MAPK as shown for thermosensory adaptation (Kobayashi et al., 2016). Important next steps for understanding the influence of CMK-1 on learning will be to discover additional proteins phosphorylated by CMK-1 and to analyze their contribution to behavioral plasticity.

In conclusion, we have characterized the ability of $c m k-1$ to profoundly affect learning in C. elegans. This, along with the sufficiency of this gene in a single pair of neurons to rescue this 
behavior, illustrates a relatively simple circuit in this animal to adapt to the natural world. The fact that worm CMK-1 is thought to serve functions performed by two different proteins in mammals, CaMKII and CaMKI/CaMKIV (Cohen et al., 2015), speaks further to the efficiency of this system. Evolutionary advantages of such a model include the ability to perform relatively complex behaviors with very few neurons and molecular players. Potential risks, however, may include insufficient compensatory mechanisms to rescue the system when damaged. The ability to learn to avoid areas of high salt upon starvation likely evolved as a way for organisms to minimize energy exploring barren areas and instead seek food-rich habitats.

\section{References}

Adachi T, Kunitomo H, Tomioka M, Ohno H, Okochi Y, Mori I, Iino Y (2010) Reversal of salt preference is directed by the insulin/PI3K and Gq/PKC signaling in Caenorhabditis elegans. Genetics 186:1309-1319. CrossRef Medline

Ardiel EL, Rankin CH (2010) An elegant mind: learning and memory in Caenorhabditis elegans. Learn Mem 17:191-201. CrossRef Medline

Bargmann CI, Horvitz HR (1991) Chemosensory neurons with overlapping functions direct chemotaxis to multiple chemicals in C. elegans. Neuron 7:729-742. CrossRef Medline

Biron D, Wasserman S, Thomas JH, Samuel AD, Sengupta P (2008) An olfactory neuron responds stochastically to temperature and modulates Caenorhabditis elegans thermotactic behavior. Proc Natl Acad Sci U S A 105:11002-11007. CrossRef Medline

Chalasani SH, Chronis N, Tsunozaki M, Gray JM, Ramot D, Goodman MB, Bargmann CI (2007) Dissecting a circuit for olfactory behaviour in Caenorhabditis elegans. Nature 450:63-70. CrossRef Medline

Choe KP (2013) Physiological and molecular mechanisms of salt and water homeostasis in the nematode Caenorhabditis elegans. Am J Physiol Regul Integr Comp Physiol 305:R175-R186. CrossRef Medline

Chronis N, Zimmer M, Bargmann CI (2007) Microfluidics for in vivo imaging of neuronal and behavioral activity in Caenorhabditis elegans. Nat Methods 4:727-731. CrossRef Medline

Clark DA, Biron D, Sengupta P, Samuel AD (2006) The AFD sensory neurons encode multiple functions underlying thermotactic behavior in Caenorhabditis elegans. J Neurosci 26:7444-7451. CrossRef Medline

Cohen SM, Li B, Tsien RW, Ma H (2015) Evolutionary and functional perspectives on signaling from neuronal surface to nucleus. Biochem Biophys Res Commun 460:88-99. CrossRef Medline

Eto K, Takahashi N, Kimura Y, Masuho Y, Arai K, Muramatsu MA, Tokumitsu $\mathrm{H}$ (1999) $\mathrm{Ca}^{2+} /$ calmodulin-dependent protein kinase cascade in Caenorhabditis elegans implication in transcriptional activation. J Biol Chem 274:22556-22562. CrossRef Medline

Evans DG, Birch JM, Ramsden RT, Sharif S, Baser ME (2006) Malignant transformation and new primary tumours after therapeutic radiation for benign disease: substantial risks in certain tumour prone syndromes. J Med Genet 43:289-294. CrossRef Medline

Evans TC (2006) Transformation and microinjection. In: The C. elegans Research Community (Ambros V, ed). Aurora, CO: WormBook. CrossRef

Fehlauer H, Nekimken AL, Kim AA, Pruitt BL, Goodman MB, Krieg M (2018) Using a microfluidics device for mechanical stimulation and high resolution imaging of C. elegans. J Vis Exp 132:e56530. CrossRef Medline

Geerling JC, Loewy AD (2008) Central regulation of sodium appetite. Exp Physiol 93:177-209. CrossRef Medline

Goodman MB, Hall DH, Avery L, Lockery SR (1998) Active currents regulate sensitivity and dynamic range in C. elegans neurons. Neuron 20:763772. CrossRef Medline

Hobert O (2014) Development of left/right asymmetry in the Caenorhabditis elegans nervous system: from zygote to postmitotic neuron. Genesis 52:528543. CrossRef Medline

Holmberg CI, Hietakangas V, Mikhailov A, Rantanen JO, Kallio M, Meinander A, Hellman J, Morrice N, MacKintosh C, Morimoto RI, Eriksson JE, Sistonen L (2001) Phosphorylation of serine 230 promotes inducible transcriptional activity of heat shock factor 1. EMBO J 20:3800-3810. CrossRef Medline

Hook SS, Means AR (2001) $\mathrm{Ca}^{2+} / \mathrm{CaM}$-dependent kinases: from activation to function. Annu Rev Pharmacol Toxicol 41:471-505. CrossRef Medline
Hostettler L, Grundy L, Käser-Pébernard S, Wicky C, Schafer WR, Glauser DA (2017) The bright fluorescent protein $\mathrm{mNeonGreen} \mathrm{facilitates} \mathrm{pro-}$ tein expression analysis in vivo. G3 7:607-615. CrossRef Medline

Hukema RK, Rademakers S, Dekkers MP, Burghoorn J, Jansen G (2006) Antagonistic sensory cues generate gustatory plasticity in Caenorhabditis elegans. EMBO J 25:312-322. CrossRef Medline

Hukema RK, Rademakers S, Jansen G (2008) Gustatory plasticity in C. elegans involves integration of negative cues and $\mathrm{NaCl}$ taste mediated by serotonin, dopamine, and glutamate. Learn Mem 15:829-836. CrossRef Medline

Hurley SW, Johnson AK (2015) The biopsychology of salt hunger and sodium deficiency. Pflugers Arch 467:445-456. CrossRef Medline

Ino Y, Yoshida K (2009) Parallel use of two behavioral mechanisms for chemotaxis in Caenorhabditis elegans. J Neurosci 29:5370-5380. CrossRef Medline

Ikeda DD, Duan Y, Matsuki M, Kunitomo H, Hutter H, Hedgecock EM, Iino Y (2008) CASY-1, an ortholog of calsyntenins/alcadeins, is essential for learning in Caenorhabditis elegans. Proc Natl Acad Sci U S A 105:52605265. CrossRef Medline

Ishihara T, Iino Y, Mohri A, Mori I, Gengyo-Ando K, Mitani S, Katsura I (2002) HEN-1, a secretory protein with an LDL receptor motif, regulates sensory integration and learning in Caenorhabditis elegans. Cell 109:639_ 649. CrossRef Medline

Iwata R, Oda S, Kunitomo H, Iino Y (2011) Roles for class IIA phosphatidylinositol transfer protein in neurotransmission and behavioral plasticity at the sensory neuron synapses of Caenorhabditis elegans. Proc Natl Acad Sci U S A 108:7589-7594. CrossRef Medline

Jansen G, Weinkove D, Plasterk RH (2002) The G-protein gamma subunit $g p c-1$ of the nematode C. elegans is involved in taste adaptation. EMBO J 21:986-994. CrossRef Medline

Kano T, Brockie PJ, Sassa T, Fujimoto H, Kawahara Y, Iino Y, Mellem JE, Madsen DM, Hosono R, Maricq AV (2008) Memory in Caenorhabditis elegans is mediated by NMDA-type ionotropic glutamate receptors. Curr Biol 18:1010-1015. CrossRef Medline

Kauffman AL, Ashraf JM, Corces-Zimmerman MR, Landis JN, Murphy CT (2010) Insulin signaling and dietary restriction differentially influence the decline of learning and memory with age. PLoS Biol 8:e1000372. CrossRef Medline

Kim KW, Jin Y (2015) Neuronal responses to stress and injury in C. elegans. FEBS Lett 589:1644-1652. CrossRef Medline

Kimura Y, Corcoran EE, Eto K, Gengyo-Ando K, Muramatsu MA, Kobayashi R, Freedman JH, Mitani S, Hagiwara M, Means AR, Tokumitsu H (2002) A CaMK cascade activates CRE-mediated transcription in neurons of Caenorhabditis elegans. EMBO Rep 3:962-966. CrossRef Medline

Kobayashi K, Nakano S, Amano M, Tsuboi D, Nishioka T, Ikeda S, Yokoyama G, Kaibuchi K, Mori I (2016) Single-cell memory regulates a neural circuit for sensory behavior. Cell Rep 14:11-21. CrossRef Medline

Kunitomo H, Sato H, Iwata R, Satoh Y, Ohno H, Yamada K, Iino Y (2013) Concentration memory-dependent synaptic plasticity of a taste circuit regulates salt concentration chemotaxis in Caenorhabditis elegans. Nat Commun 4:2210. CrossRef Medline

Kuramochi M, Doi M (2017) A computational model based on multiregional calcium imaging represents the spatio-temporal dynamics in a Caenorhabditis elegans sensory neuron. PLoS One 12:e0168415. CrossRef Medline

Luo L, Wen Q, Ren J, Hendricks M, Gershow M, Qin Y, Greenwood J, Soucy ER, Klein M, Smith-Parker HK, Calvo AC, Colón-Ramos DA, Samuel AD, Zhang Y (2014) Dynamic encoding of perception, memory, and movement in a C. elegans chemotaxis circuit. Neuron 82:1115-1128. CrossRef Medline

Matsuki M, Kunitomo H, Iino Y (2006) Goalpha regulates olfactory adaptation by antagonizing $\mathrm{G}_{\mathrm{q}} \alpha$-DAG signaling in Caenorhabditis elegans. Proc Natl Acad Sci U S A 103:1112-1117. CrossRef Medline

Miyabayashi T, Palfreyman MT, Sluder AE, Slack F, Sengupta P (1999) Expression and function of members of a divergent nuclear receptor family in Caenorhabditis elegans. Dev Biol 215:314-331. CrossRef Medline

Moss BJ, Park L, Dahlberg CL, Juo P (2016) The CaM kinase CMK-1 mediates a negative feedback mechanism coupling the C. elegans glutamate receptor GLR-1 with its own transcription. PLoS Genet 12:e1006180. CrossRef Medline

Neal SJ, Takeishi A, O’Donnell MP, Park J, Hong M, Butcher RA, Kim K, Sengupta P (2015) Feeding state-dependent regulation of developmen- 
tal plasticity via CaMKI and neuroendocrine signaling. eLife 4:e11547. CrossRef Medline

Nekimken AL, Fehlauer H, Kim AA, Manosalvas-Kjono SN, Ladpli P, Memon F, Gopisetty D, Sanchez V, Goodman MB, Pruitt BL, Krieg M (2017) Pneumatic stimulation of C. elegans mechanoreceptor neurons in a microfluidic trap. Lab Chip 17:1116-1127. CrossRef Medline

Oda S, Tomioka M, Ino Y (2011) Neuronal plasticity regulated by the insulin-like signaling pathway underlies salt chemotaxis learning in Caenorhabditis elegans. J Neurophysiol 106:301-308. CrossRef Medline

Ortiz CO, Faumont S, Takayama J, Ahmed HK, Goldsmith AD, Pocock R, McCormick KE, Kunimoto H, Iino Y, Lockery S, Hobert O (2009) Lateralized gustatory behavior of $C$. elegans is controlled by specific receptortype guanylyl cyclases. Curr Biol 19:996-1004. CrossRef Medline

Rabinowitch I, Chatzigeorgiou M, Zhao B, Treinin M, Schafer WR (2014) Rewiring neural circuits by the insertion of ectopic electrical synapses in transgenic C. elegans. Nat Commun 5:4442. CrossRef Medline

Ramot D, Johnson BE, Berry TL Jr, Carnell L, Goodman MB (2008) The parallel worm tracker: a platform for measuring average speed and druginduced paralysis in nematodes. PLoS One 3:e2208. CrossRef Medline

Saeki S, Yamamoto M, Iino Y (2001) Plasticity of chemotaxis revealed by paired presentation of a chemoattractant and starvation in the nematode Caenorhabditis elegans. J Exp Biol 204:1757-1764. Medline

Sakai N, Iwata R, Yokoi S, Butcher RA, Clardy J, Tomioka M, Iino Y (2013) A sexually conditioned switch of chemosensory behavior in C. elegans. PLoS One 8:e68676. CrossRef Medline

Sakashita T, Hamada N, Ikeda DD, Yanase S, Suzuki M, Ishii N, Kobayashi Y (2008) Modulatory effect of ionizing radiation on food- $\mathrm{NaCl}$ associative learning: the role of gamma subunit of $\mathrm{G}$ protein in Caenorhabditis elegans. FASEB J 22:713-720. CrossRef Medline

Satterlee JS, Ryu WS, Sengupta P (2004) The CMK-1 CaMKI and the TAX-4 cyclic nucleotide-gated channel regulate thermosensory neuron gene expression and function in C. elegans. Curr Biol 14:62-68. CrossRef Medline

Schild LC, Zbinden L, Bell HW, Yu YV, Sengupta P, Goodman MB, Glauser DA (2014) The balance between cytoplasmic and nuclear CaM kinase-1 signaling controls the operating range of noxious heat avoidance. Neuron 84:983-996. CrossRef Medline

Schindelin J, Arganda-Carreras I, Frise E, Kaynig V, Longair M, Pietzsch T,
Preibisch S, Rueden C, Saalfeld S, Schmid B, Tinevez JY, White DJ, Hartenstein V, Eliceiri K, Tomancak P, Cardona A (2012) Fiji: an opensource platform for biological-image analysis. Nat Methods 9:676-682. CrossRef Medline

Sheng M, Thompson MA, Greenberg ME (1991) CREB: a Ca ${ }^{2+}$-regulated transcription factor phosphorylated by calmodulin-dependent kinases. Science 252:1427-1430. CrossRef Medline

Stetak A, Hörndli F, Maricq AV, van den Heuvel S, Hajnal A (2009) Neuron-specific regulation of associative learning and memory by MAGI-1 in C. elegans. PLoS One 4:e6019. CrossRef Medline

Suzuki H, Thiele TR, Faumont S, Ezcurra M, Lockery SR, Schafer WR (2008) Functional asymmetry in Caenorhabditis elegans taste neurons and its computational role in chemotaxis. Nature 454:114-117. CrossRef Medline

Thiele TR, Faumont S, Lockery SR (2009) The neural network for chemotaxis to tastants in Caenorhabditis elegans is specialized for temporal differentiation. J Neurosci 29:11904-11911. CrossRef Medline

Tomioka M, Adachi T, Suzuki H, Kunitomo H, Schafer WR, Iino Y (2006) The insulin/PI 3-kinase pathway regulates salt chemotaxis learning in Caenorhabditis elegans. Neuron 51:613-625. CrossRef Medline

Vellai T, McCulloch D, Gems D, Kovács AL (2006) Effects of sex and insulin/insulin-like growth factor-1 signaling on performance in an associative learning paradigm in Caenorhabditis elegans. Genetics 174:309316. CrossRef Medline

Ward S (1973) Chemotaxis by the nematode Caenorhabditis elegans: identification of attractants and analysis of the response by use of mutants. Proc Natl Acad Sci U S A 70:817-821. CrossRef Medline

Wayman GA, Lee YS, Tokumitsu H, Silva A, Soderling TR (2008) Calmodulin-kinases: modulators of neuronal development and plasticity. Neuron 59:914-931. CrossRef Medline

Yu S, Avery L, Baude E, Garbers DL (1997) Guanylyl cyclase expression in specific sensory neurons: a new family of chemosensory receptors. Proc Natl Acad Sci U S A 94:3384-3387. CrossRef Medline

Yu YV, Bell HW, Glauser D, Van Hooser SD, Goodman MB, Sengupta P (2014) CaMKI-dependent regulation of sensory gene expression mediates experience-dependent plasticity in the operating range of a thermosensory neuron. Neuron 84:919-926. CrossRef Medline 